\title{
ORBITAL AND PHYSICAL PROPERTIES OF THE $\sigma$ Ori Aa, Ab, B TRIPLE SYSTEM
}

\author{
S. Simón-Díaz ${ }^{1,2}$, J. A. Caballero ${ }^{3}$, J. Lorenzo ${ }^{4}$, J. Maíz Apellániz ${ }^{3,5}$, F. R. N. Schneider ${ }^{6}$, I. Negueruela ${ }^{4}$, \\ R. H. Barbá ${ }^{7}$, R. Dorda ${ }^{4}$, A. Marco ${ }^{4}$, D. Montes ${ }^{8}$, A. Pellerin ${ }^{9}$, J. Sanchez-Bermudez ${ }^{5}$, Á. Sódor ${ }^{10}$, and A. Sota ${ }^{5}$ \\ ${ }^{1}$ Instituto de Astrofísica de Canarias, E-38200 La Laguna, Tenerife, Spain; ssimon@ @iac.es \\ 2 Departamento de Astrofísica, Universidad de La Laguna, E-38205 La Laguna, Tenerife, Spain \\ ${ }^{3}$ Centro de Astrobiología (CSIC-INTA), ESAC Campus, P.O. Box 78, E-28691 Villanueva de la Cañada, Madrid, Spain \\ ${ }^{4}$ Departamento de Física, Ingeniería de Sistemas y Teoría de la Señal, Escuela Politécnica Superior, University of Alicante, Apdo. 99 , E-03080 Alicante, Spain \\ ${ }_{5}^{5}$ Instituto de Astrofísica de Andalucía (CSIC), Glorieta de la Astronomía s/n, E-18008 Granada, Spain \\ ${ }^{6}$ Argelander-Institut für Astronomie der Universität Bonn, Auf dem Hügel 71, D-53121 Bonn, Germany \\ ${ }^{7}$ Departamento de Física, Universidad de La Serena, Benavente 980, La Serena, Chile \\ ${ }^{8}$ Departamento Astrofísica, Facultad de Ciencias Físicas, Universidad Complutense de Madrid, E-28040 Madrid, Spain \\ ${ }^{9}$ Department of Physics and Astronomy, State University of New York at Geneseo, 1 College Circle, Geneseo, NY 14454, USA \\ ${ }^{10}$ Konkoly Observatory, Research Centre for Astronomy and Earth Sciences, Hungarian Academy of Sciences, H-01121 Budapest, Hungary \\ Received 2014 October 22; accepted 2014 December 10; published 2015 January 27
}

\begin{abstract}
We provide a complete characterization of the astrophysical properties of the $\sigma$ Ori Aa, Ab, B hierarchical triple system and an improved set of orbital parameters for the highly eccentric $\sigma$ Ori Aa, Ab spectroscopic binary. We compiled a spectroscopic data set comprising 90 high-resolution spectra covering a total time span of 1963 days. We applied the Lehman-Filhés method for a detailed orbital analysis of the radial velocity curves and performed a combined quantitative spectroscopic analysis of the $\sigma$ Ori Aa, Ab, B system by means of the stellar atmosphere code FASTWIND. We used our own plus other available information on photometry and distance to the system for measuring the radii, luminosities, and spectroscopic masses of the three components. We also inferred evolutionary masses and stellar ages using the Bayesian code BONNSAI. The orbital analysis of the new radial velocity curves led to a very accurate orbital solution of the $\sigma$ Ori Aa, Ab pair. We provided indirect arguments indicating that $\sigma$ Ori B is a fast-rotating early B dwarf. The FASTWIND+BONNSAI analysis showed that the Aa, Ab pair contains the hottest and most massive components of the triple system while $\sigma$ Ori B is a bit cooler and less massive. The derived stellar ages of the inner pair are intriguingly younger than the one widely accepted for the $\sigma$ Orionis cluster, at $3 \pm 1 \mathrm{Ma}$. The outcome of this study will be of key importance for a precise determination of the distance to the $\sigma$ Orionis cluster, the interpretation of the strong X-ray emission detected for $\sigma$ Ori $\mathrm{Aa}, \mathrm{Ab}$, $\mathrm{B}$, and the investigation of the formation and evolution of multiple massive stellar systems and substellar objects.
\end{abstract}

Key words: binaries: spectroscopic - open clusters and associations: individual (sigma Orionis) stars: early-type - stars: individual (sigma Ori) - stars: massive

\section{INTRODUCTION}

The fourth-brightest star in Orion's Belt is $\sigma$ Ori (Quce ultimam baltei pracedit ad austrum, 48 Ori, HD 37648). This Trapezium-like system is also the brightest source of the relatively nearby, almost extinction-free, $\sim 3$ Ma old $\sigma$ Orionis open cluster in the Ori OB1b association, which is widely acknowledged as a cornerstone for the study of stellar and substellar formation (Garrison 1967; Wolk 1996; Béjar et al. 1999; Walter et al. 2008; Caballero 2008b, 2013). Remarkably, the components in the eponymous $\sigma$ Ori stellar system illuminate and shape the celebrated, conspicuous Horsehead Nebula (Pety et al. 2005; Habart et al. 2005; Goicoechea et al. 2006, 2009; Compiègne et al. 2007; Rimmer et al. 2012) and a close photoeroded pair of a very low mass star and a brown dwarf proplyd (van Loon \& Oliveira 2003; Sanz-Forcada et al. 2004; Caballero 2005; Bouy et al. 2009; Hodapp et al. 2009). Therefore, the amount of high-energy photons injected by the high-mass $\sigma$ Ori stars into the intracluster medium is a compulsory input not only for testing certain models of low-mass star and brown dwarf formation (e.g., Whitworth et al. 2007) but also for understanding the astrochemistry in the Horsehead photodissociation region (Abergel et al. 2003; Pound et al. 2003; Ward-Thompson et al. 2006; Bowler et al. 2009, and references above).

Currently, we know that there are five stars with spectral types earlier than B3 (i.e., massive stars) in the central arcminute of the cluster, including $\sigma$ Ori Aa, Ab, B, D, and E (Caballero 2014).
Contrarily to $\sigma$ Ori $\mathrm{D}$, which is a normal $\mathrm{B} 2 \mathrm{~V}$ star with large projected rotational velocity ( $v \sin i=200 \mathrm{~km} \mathrm{~s}^{-1}$; Simón-Díaz \& Herrero 2014), the other four stars have extensively caught the attention of the stellar community. In particular, $\sigma$ Ori $\mathrm{E}$ is a famous helium-rich, magnetically strong, peculiar, variable star with spectral type B2Vp (Walborn 1974; Landstreet \& Borra 1978; Groote \& Hunger 1997; Townsend et al. 2013), while $\sigma$ Ori $\mathrm{Aa}, \mathrm{Ab}, \mathrm{B}$ is a very high mass, hierarchical triple system. It is made of a $\sim 0$ !'25-wide astrometric binary, "Aa, Ab-B," which has not completed a full revolution yet since its discovery $\left(P_{\text {astrom }}=156.7 \pm 3.0\right.$ a; Burnham 1892; Turner et al. 2008; Caballero 2008a, 2014), and a spectroscopic binary, "Aa-Ab," in a highly eccentric orbit with a period 400 times smaller than the astrometric period $\left(P_{\mathrm{SB} 2}=143.5 \pm 0.5\right.$ days; Frost \& Adams 1904; Miczaika 1950; Bolton 1974; Morrell \& Levato 1991; Stickland \& Lloyd 2001; Simón-Díaz et al. 2011a).

This paper aims to be a continuation of the study started by Simón-Díaz et al. (2011a, Paper I), who compiled 23 Fiber-fed Echelle Spectrograph (FIES) spectra between 2008 November and 2011 April, confirmed the presence of a third massive star component in the $\sigma$ Ori $\mathrm{AB}$ system, and determined for the first time the orbital parameters of the $\mathrm{Aa}, \mathrm{Ab}$ pair. Here we present the analysis of a much-extended data set comprising a total of 90 high-resolution spectra spanning $\sim 5 \mathrm{yr}$. Besides the refinement of the orbital parameters determined from a longer and much better sampled radial velocity curve, we perform a quantitative spectroscopic analysis of the combined spectra of $\sigma$ 
Ori Aa, Ab, B. The derived spectroscopic parameters (effective temperatures, gravities, and projected rotational velocities) are complemented with photometric information on the system to provide the complete set of stellar astrophysical parameters, namely, radii, luminosities, spectroscopic and evolutionary masses, plus estimates of the stellar ages and number of ionizing photons. The paper, which partially benefits from preliminary results from recent interferometric observations by Schaefer (2013) and Hummel et al. (2013), concludes with a discussion on the global orbital and physical properties of the $\sigma$ Ori Aa, $\mathrm{Ab}$, B system, highlighting the importance of the results of our study for the interpretation of the strong X-ray emission of the triple system, a precise determination of the distance to the $\sigma$ Orionis cluster, and new observational clues in the investigation of the formation and evolution of stars and brown dwarfs at all mass domains.

\section{OBSERVATIONS}

After publication of Paper I, we continued gathering highresolution optical spectra with five different instruments, mainly attached to medium-sized telescopes. Our final sample of 90 spectra covers a total time span of 1963 days, equivalent to almost 14 orbital periods of the Aa, Ab system. In particular, we could obtain over one-third of the spectra near the periastron passages of 2010 September, 2011 November, 2012 April, 2013 October/November, and 2014 March.

1. Fifteen epochs were obtained with the FIES spectrograph $(R=46,000)$ at the $2.5 \mathrm{~m}$ Nordic Optical Telescope at El Roque de los Muchachos Observatory as part of the IACOB project (Simón-Díaz et al. 2011b, 2011d). Together with the spectra used in Paper I, these represent a total of 38 epochs.

2. Thirty epochs were obtained with the High Efficiency and Resolution Mercator Echelle Spectrograph (HERMES) $(R=85,000)$ at the $1.2 \mathrm{~m}$ MERCATOR telescope at El Roque de los Muchachos Observatory, also as part of the IACOB project.

3. Eleven epochs were obtained with the Calar Alto Fiber-fed Echelle (CAFÉ) spectrograph $(R=65,000)$ at the $2.2 \mathrm{~m}$ telescope at Calar Alto Observatory as part of the CAFÉBEANS project (Negueruela et al. 2014).

4. Six epochs were obtained with the High Resolution Spectrograph (HRS) $(R=30,000)$ at the $9.2 \mathrm{~m}$ Hobby-Eberly Telescope as part of the NoMaDS project (Maíz Apellániz et al. 2012).

5. In addition, we included in our data set four spectra obtained with the Fiberfed Extended Range Optical Spectrograph (FEROS) $(R=48,000)$ at the $2.2 \mathrm{~m}$ telescope at La Silla Observatory as part of the OWN survey (Barbá et al. 2010).

Full descriptions of the various used instruments can be found in Telting et al. (2014, FIES), Raskin et al. (2011; HERMES), Aceituno et al. (2013; CAFÉ), Tull (1998; HRS), and Kaufer et al. (1999; FEROS). The log of the observations is presented in Table A1, while Figure 1 shows portions of $\sigma$ Ori Aa, Ab, B spectra at seven representative epochs. The typical signal-tonoise ratio $(\mathrm{S} / \mathrm{N})$ of all the spectra was above 200 , which could be reached with exposure times of less than 10 minutes in all telescopes. In the case of the two HERMES spectra obtained on 2013 October 31 (on the exact date of the closest quadrature to periastron passage), we increased the exposure time to reach an $\mathrm{S} / \mathrm{N} \sim 350$.

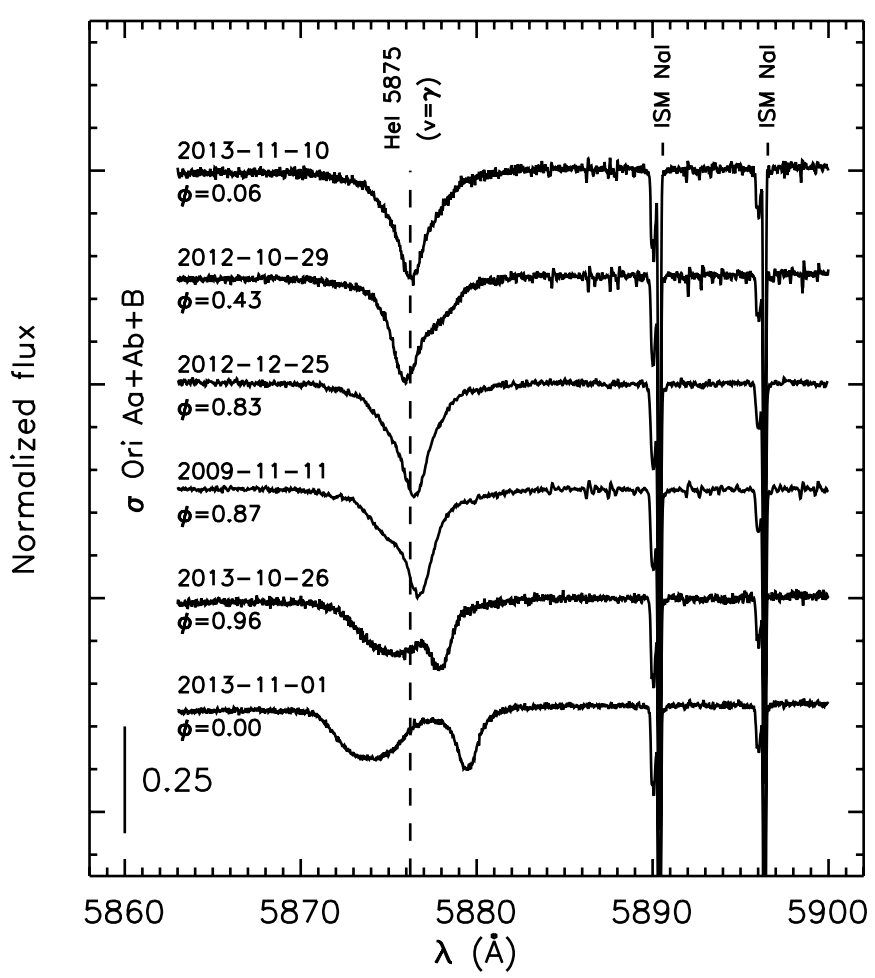

Figure 1. Spectra of $\sigma$ Ori Aa, Ab, B in the region of the stellar $\mathrm{He}$ I $\lambda 5875$ and interstellar $\mathrm{Na}$ I lines at seven representative epochs. The vertical dashed line indicates the wavelength of the He I line shifted to the systemic velocity $(\gamma=$ $+31.10 \mathrm{~km} \mathrm{~s}^{-1}$ ). All spectra were corrected from telluric lines.

The FIES, HERMES, and FEROS spectra were reduced using the corresponding available pipelines (FIEStool ${ }^{11}$, Hermes$\mathrm{DRS}^{12}$, and FEROS-DRS ${ }^{13}$, respectively). We used our own pipelines for reducing the CAFÉ and HET spectra. In all cases we used the information provided in the corresponding headers of the fits files to correct the spectra for heliocentric velocity and our own routines implemented in IDL for continuum normalization.

We also obtained lucky imaging of the $\sigma$ Ori Aa, Ab, B system with the AstraLux instrument at the Calar Alto $2.2 \mathrm{~m}$ telescope (Hormuth et al. 2008). We used three different filters (Sloan $i$ and $z$ and a narrowband filter centered at $9137 \AA$ ) at five different epochs (from 2008 January to 2013 September; see Table 2). The seeing varied between 0.9 and 1".3, but component B was clearly separated from $A$ in the processed data in all cases (Aa and $\mathrm{Ab}$ components cannot be separated with lucky imaging). The first epoch was discussed by Maíz Apellániz (2010), where the reader is referred for further details on the data.

\section{ANALYSIS AND RESULTS}

\subsection{Radial Velocity}

We followed a similar strategy to that described in Paper I in revisiting the orbital parameters of the $\mathrm{Aa}, \mathrm{Ab}$ system resulting from the analysis of the extended radial velocity curves. In brief, we first used the He I $\lambda 5875$ line to determine the radial velocity of the two components. To this aim, we performed a two-parameter cross-correlation of the observed spectra with a grid of synthetic spectra built with two rotationally broadened,

\footnotetext{
11 http://www.not.iac.es/instruments/fies/fiestool/FIEStool.html

12 http://www.mercator.iac.es/instruments/hermes/hermesdrs.php

13 http://www.eso.org/sci/facilities/lasilla/instruments/feros/tools/DRS.html
} 


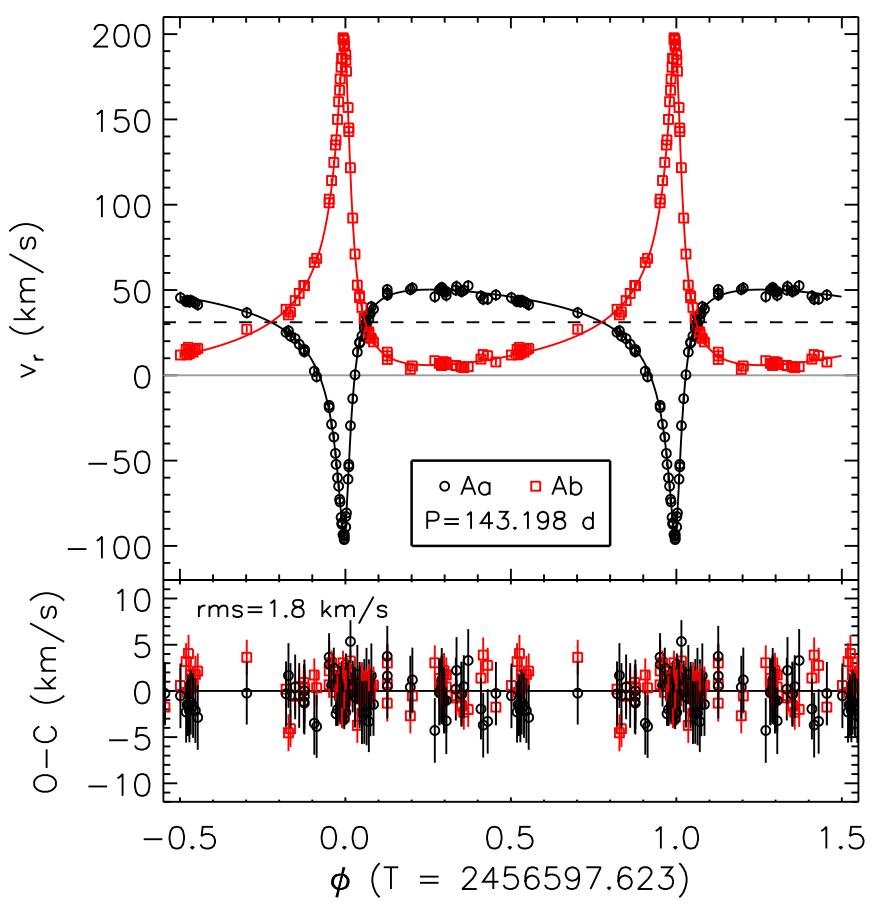

Figure 2. Upper panel: radial velocity curves of $\sigma$ Ori Aa (black circles) and Ab (red squares) phased to the period $P=143.198$ days. Error bar sizes are smaller than those of symbol size. The horizontal dashed line indicates the systemic velocity (see Table 1). Lower panel: velocity residuals to the adopted fit for the two components. The root-mean-square of the fit is $1.8 \mathrm{~km} \mathrm{~s}^{-1}$.

Table 1

Revised Orbital Parameters of the $\sigma$ Ori Aa, Ab System

\begin{tabular}{|c|c|c|c|}
\hline Parameter & \multicolumn{2}{|c|}{ Value } & Unit \\
\hline$P_{\mathrm{Aa}, \mathrm{Ab}}$ & \multicolumn{2}{|c|}{$143.198 \pm 0.005$} & days \\
\hline$T$ & \multicolumn{2}{|c|}{$2456597.623 \pm 0.024$} & days \\
\hline$e$ & \multicolumn{2}{|c|}{$0.7782 \pm 0.0011$} & \\
\hline$\gamma$ & \multicolumn{2}{|c|}{$+31.10 \pm 0.16$} & $\mathrm{~km} \mathrm{~s}^{-1}$ \\
\hline$\omega$ & \multicolumn{2}{|c|}{$199.98 \pm 0.24$} & deg \\
\hline \multirow[t]{2}{*}{$M_{\mathrm{Aa}} / M_{\mathrm{Ab}}$} & \multicolumn{2}{|c|}{$1.325 \pm 0.006$} & \\
\hline & Aa & $\mathrm{Ab}$ & \\
\hline$K$ & $71.9 \pm 0.3$ & $95.2 \pm 0.3$ & $\mathrm{~km} \mathrm{~s}^{-1}$ \\
\hline$a \sin i$ & $127.7 \pm 0.6$ & $169.2 \pm 0.6$ & $R_{\odot}$ \\
\hline$M \sin ^{3} i$ & $9.78 \pm 0.07$ & $7.38 \pm 0.05$ & $M_{\odot}$ \\
\hline
\end{tabular}

radial-velocity-shifted, FASTWIND He I line profiles. We used $v \sin i=135$ and $35 \mathrm{~km} \mathrm{~s}^{-1}$ for the Aa and $\mathrm{Ab}$ components, respectively (Stickland \& Lloyd 2001; Simón-Díaz et al. 2011a). The measured radial velocities, along with their corresponding uncertainties associated with the cross-correlations, are given in Table A1.

Next, we applied the Lehmann-Filhés method implemented in $\mathrm{SBOP}^{14}$ (Etzel 2004) for a detailed orbital analysis of the radial velocity curves. We assumed as initial parameters those values indicated in Table 2 of Paper I. Contrarily to our previous analysis, now we consider the period as a free parameter to be determined by SBOP. The resulting radial velocity curves, phased to the derived period, are shown in Figure 2, and the revised orbital parameters are provided in Table 1.

The agreement with the solution presented in Paper I is very good (but note the $180 \mathrm{deg}$ indetermination of $\omega$ in Paper I). However, as a consequence of the larger time span and the better phase coverage (especially around periastron passage) of

\footnotetext{
14 http://mintaka.sdsu.edu/faculty/etzel/
}

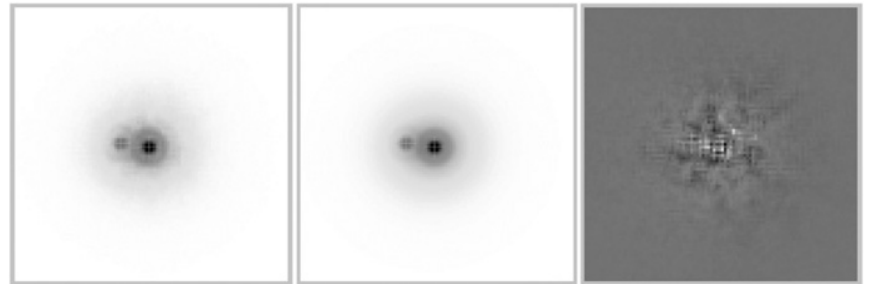

Figure 3. AstraLux observations of $\sigma$ Ori A, B obtained on 2012 October 2 with the $9137 \AA$ A narrowband filter. The three panels show the original data (left), PSF fit (center), and fit residual (right). The intensity scale is linear in the three cases, with the range corresponding to $0 \%-80 \%$ of the maximum data pixel in the first two panels and to $-5 \%$ to $+5 \%$ of the maximum data pixel in the third one. Each panel is about 2 .'5 on each side. North is up, and east is left.

the new radial velocity curves, we have improved in one order of magnitude the accuracy of all the resulting quantities. In particular, the period has been refined from $143.5 \pm 0.5$ days to $143.198 \pm 0.005$ days. The new, more accurate, systemic velocity of the $\sigma$ Ori Aa, Ab system $\left(+31.10 \pm 0.16 \mathrm{~km} \mathrm{~s}^{-1}\right)$ is also in very good agreement with the systemic velocity of the cluster as determined by Sacco et al. (2008) from single low-mass stars in $\sigma$ Orionis $\left(+30.93 \pm 0.92 \mathrm{~km} \mathrm{~s}^{-1}\right)$. The mass ratio of the two components has increased from $1.23 \pm 0.07$ to $1.325 \pm 0.006$. The projected semimajor axes and masses have also been slightly modified.

\subsection{Photometry}

The $V$ magnitude of $\sigma$ Ori, including the three components, is 3.80 mag (Johnson et al. 1966; Lee 1968; Vogt 1976; Ducati et al. 2001). However, the determination of radii, luminosities, and masses of each component in Section 3.4.2 requires the individual absolute magnitudes in the $V$ band. Also, this information is of interest for double-checking the dilution factors considered in the combined spectroscopic analysis in Section 3.4.1. For obtaining the extinction-corrected $V$-band absolute magnitudes of the three components we also need information about the magnitude difference between the A, B and Aa, Ab pairs, along with the extinction and distance to the stars.

\subsubsection{Magnitude Difference between $\sigma$ Ori $A$ and $B$}

The difference in magnitude between the A (actually Aa, $\mathrm{Ab}$ ) and $\mathrm{B}$ components has been measured by several authors using different techniques. With Hipparcos data, Perryman et al. (1997) tabulated a magnitude difference $\Delta H_{p}=1.21 \pm$ 0.05 mag. Using adaptive optics, ten Brummelaar et al. (2000) measured the magnitude difference in three different optical filters $(\Delta V=1.24 \pm 0.10, \Delta R=1.34 \pm 0.13$, and $\Delta I=$ $1.25 \pm 0.15 \mathrm{mag}$ ). Later on, Horch et al. (2001) provided four speckle $V$-band differential photometry measures for $\sigma$ Ori A, $\mathrm{B}$ ranging from 1.05 to $1.44 \mathrm{mag}$ (with a mean value of $1.18 \pm$ $0.08 \mathrm{mag}$ ). Finally, Horch et al. (2004) obtained again new speckle observations more consistent with previous adaptive optics measurements $\left(\Delta m_{503 \mathrm{~nm}}=1.30 \pm 0.07, \Delta m_{648 \mathrm{~nm}}=\right.$ $\left.1.25 \pm 0.03, \Delta m_{701 \mathrm{~nm}} \sim 1.26 \mathrm{mag}\right)$.

We measured magnitude differences, angular separations $(\rho)$, and position angles $(\theta)$ in our own lucky imaging data (Section 2). The AstraLux images were processed using the strategy described in Maíz Apellániz (2010) but with one important difference: instead of using a two-dimensional Gaussian for the point-spread function (PSF) core, we used an obstructed Airy pattern with the parameters of the Calar Alto $2.2 \mathrm{~m}$ telescope convolved with a two-dimensional Gaussian. An example of the data and fits is shown in Figure 3, while Table 2 provides our measurements. 
Table 2

Relative Astrometry and Photometry of $\sigma$ Ori A, B from the AstraLux Data

\begin{tabular}{lcccc}
\hline \hline $\begin{array}{l}\text { Date } \\
\text { (yyyy mm dd) }\end{array}$ & Filter & $\begin{array}{c}\rho \\
(\mathrm{mas})\end{array}$ & $\begin{array}{c}\theta \\
(\mathrm{deg})\end{array}$ & $\begin{array}{c}m_{\mathrm{B}}-m_{\mathrm{A}} \\
(\mathrm{mag})\end{array}$ \\
\hline 2008 Jan 17 & $z$ & $253.9 \pm 2.0$ & $92.9 \pm 0.6$ & $1.319 \pm 0.035$ \\
2011 Sep 13 & $z$ & $254.4 \pm 1.1$ & $85.6 \pm 0.3$ & $1.183 \pm 0.019$ \\
2012 Oct 2 & $9137 \AA$ & $257.3 \pm 1.3$ & $82.6 \pm 0.3$ & $1.195 \pm 0.025$ \\
2012 Oct 3 & $i$ & $256.8 \pm 0.9$ & $82.6 \pm 0.3$ & $1.201 \pm 0.016$ \\
2013 Sep 16 & $i$ & $252.6 \pm 1.0$ & $80.1 \pm 0.4$ & $1.163 \pm 0.016$ \\
\hline
\end{tabular}

The dispersion of all magnitude differences, from 5030 to $9137 \AA$, is probably a consequence of the difficulty to characterize the PSF of the images and the proximity of the A and B components (as indicated in Maíz Apellániz 2010) rather than a color effect, which is not expected at such early spectral types. We gave more weight to our and the Hipparcos measurements and considered $\Delta V_{\mathrm{A}, \mathrm{B}}=1.20 \pm 0.05 \mathrm{mag}$.

\subsubsection{Magnitude Difference between $\sigma$ Ori Aa and Ab}

The case of $\sigma$ Ori $\mathrm{Aa}$ and $\mathrm{Ab}$ is more complex, mainly because the angular separation of these two stars is so small that only interferometric observations provide enough spatial resolution to resolve both components. As a consequence, the few estimations of the $\mathrm{Aa}, \mathrm{Ab}$ magnitude difference found in the literature come from indirect arguments based on the derived spectral types of the two components, which in many cases were erroneously associated with the A and B components. In particular, from the inspection of one of his high-resolution spectrograms showing separated lines, Bolton (1974) indicated that the redshifted component $\left(\mathrm{Ab}\right.$ at $\left.+169.6 \mathrm{~km} \mathrm{~s}^{-1}\right)$ appeared to be 0.5 mag fainter than the blueshifted component (Aa at $-88 \mathrm{~km} \mathrm{~s}^{-1}$ ). Edwards (1976) derived an independent visual magnitude difference, $\Delta V_{\mathrm{Aa}, \mathrm{Ab}}=0.78 \mathrm{mag}$, after following a more quantitative strategy aimed at determining the spectral class of the individual components of visual binaries.

Only recently, interferometric observations with the Michigan Infra-Red Combiner for the CHARA Interferometer (MIRC) and the Navy Precision Optical Interferometer (NPOI) by Schaefer (2013) and Hummel et al. (2013) have been able to provide a direct measurement of the magnitude difference between Aa and Ab. Schaefer (2013) measured a flux ratio between the two components in the near-infrared $H$ band of 0.58 , which translates into a difference in magnitude of $0.59 \mathrm{mag}$ in $H$, in good agreement with the value proposed by Bolton (1974), but incompatible with the value estimated by Edwards (1976). All in all, we assumed $\Delta V_{\mathrm{Aa}, \mathrm{Ab}}=0.59 \pm 0.05 \mathrm{mag}$.

\subsubsection{Extinction}

In most published photometry, $\sigma$ Ori Aa, Ab, B appears as a single source. We collected from the literature Strömgren uvby, Tycho-2 $B_{T} V_{T}$, Johnson-Cousins $V I$, and Two Micron All Sky Survey (2MASS) $K_{s}$ photometry for the system (2MASS $J H$ and WISE photometry saturated) and processed the data using the latest version of the photometric Bayesian code CHORIZos (Maíz Apellániz 2004). It incorporates the Milky Way spectral energy distribution grid of Maíz Apellániz (2013a), the photometric calibration and zero points of Maíz Apellániz (2005a, 2006, 2007), and the family of extinction laws of Maíz Apellániz (2013b) and Maíz Apellániz et al. (2014). We fixed the photometric luminosity class to 5.0 and left four free parameters: $T_{\text {eff }}, E$ (4405-5495) (amount of extinction), $R_{5495}$ (type of extinction), and $\log d$. We fitted nine photometric bands

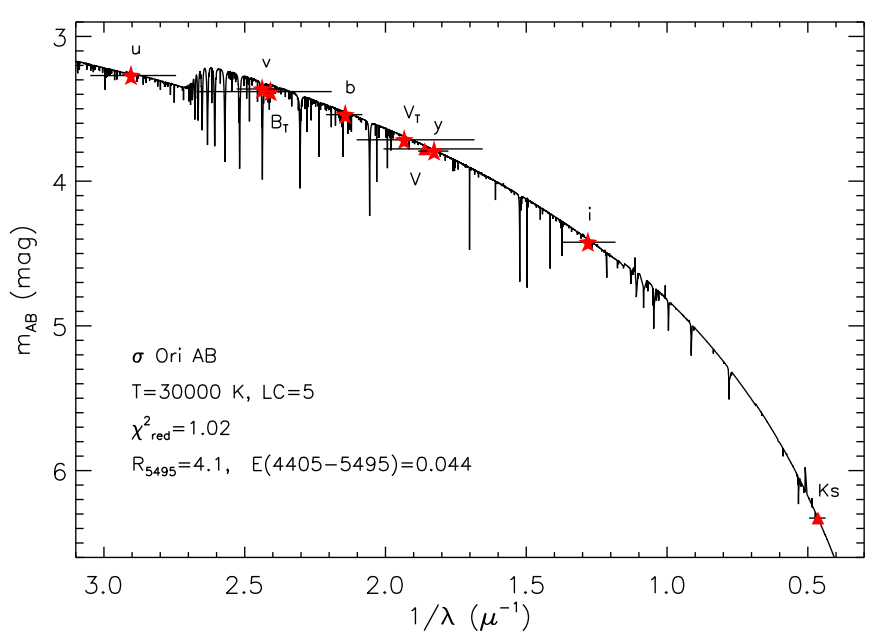

Figure 4. CHORIzos best spectral energy distribution for the $\sigma$ Ori AB photometry. Red star symbols with error bars (horizontal for filter extent, vertical for uncertainty) indicate the used photometry (Tycho-2 $B_{T} V_{T}$, 2MASS $K_{s}$, Johnson-Cousins VI, and Strömgren uvby) in the AB system.

Table 3

CHORIzos Output Parameters for $\sigma$ Ori

\begin{tabular}{lcc}
\hline \hline Parameter & Value & Unit \\
\hline$T_{*}$ & $29.9 \pm 0.6$ & $\mathrm{kK}$ \\
$E(4405-5495)$ & $0.044 \pm 0.007$ & $\mathrm{mag}$ \\
$R_{5495}$ & $4.1 \pm 0.8$ & \\
$\log d$ & $2.420 \pm 0.017$ & \\
$A_{V}$ & $0.18 \pm 0.02$ & $\mathrm{mag}$ \\
\hline
\end{tabular}

and hence had five degrees of freedom. The resulting values for these four parameters and the best spectral energy distribution are shown in Table 3 and Figure 4, respectively. The estimated extinction in the $V$ band is $A_{\mathrm{V}}=0.18 \pm 0.02 \mathrm{mag}$.

The CHORIzos execution yielded a reduced $\chi^{2}$ value of 1.0 , indicating that the photometry used is consistent and compatible with the used spectral energy distributions and extinction laws. The values of $E(4405-5495)$ and $A_{V}$ are compatible with previous results (Lee 1968; Brown et al. 1994; Mayne \& Naylor 2008). $R_{5495}$ shows a large uncertainty, as expected for an object with such a low extinction. The derived $T_{\text {eff }}$ is consistent with a composite source made out of three bright stars, one hotter than $30 \mathrm{kK}$, the other two cooler than that. Finally, the derived distance $(d \approx 263 \mathrm{pc})$ is considerably lower than other measurements, but this is just an artifact of the fitting and must not be used: since we are measuring the combined photometry of three stars and fixing the luminosity class, the resulting distance is artificially lower than the real one (see below). This problem with spectroscopic parallaxes is common for O-type systems owing to the abundance of unresolved binaries.

\subsubsection{Distance}

There have been different determinations of the distance to the $\sigma$ Orionis cluster, which have been derived and used in a variety of manners across the literature. In spite of the efforts put forth by several authors in obtaining an accurate and reliable value, there does not seem to be an acceptable consensus yet, with determinations ranging from 350 to $470 \mathrm{pc}$. Maíz Apellániz et al. (2004) gave a Lutz-Kelker-corrected value of $380_{-87}^{+136} \mathrm{pc}$ for $\sigma$ Ori based on the original Hipparcos measurement (Perryman et al. 1997), but with a self-consistent (and nonconstant) spatial distribution for the early-type stars in the solar neighborhood 
(Lutz \& Kelker 1973; Maíz Apellániz 2001, 2005b). The new Hipparcos reduction of the raw data by van Leeuwen (2007) did not improve the original measurement.

From average Hipparcos parallax measurements of stars in Ori OB1b, other authors have reported distances of up to $440 \mathrm{pc}$ (Brown et al. 1994; de Zeeuw et al. 1999; Hernández et al. 2005; Caballero \& Dinis 2008). Alternative, nonparallactic determinations of the cluster distance have been mostly based on isochronal fitting. However, perhaps because of the heterogeneity in used data and models, minimization techniques, spectral types of stars, and subjective authors' assumptions, derived distances also range on a wide interval from $360_{-60}^{+70}$ to $470 \pm 30 \mathrm{pc}$ (Sherry et al. 2004, 2008; Hernández et al. 2005; Caballero 2007; Mayne \& Naylor 2008; Naylor 2009).

A classical distance-determination method, the dynamical parallax, was implemented in the cluster by Caballero (2008a), who estimated $d \sim 385 \mathrm{pc}$ under the assumption that $\sigma$ Ori was triple. This estimation matches the recent determinations with NPOI (Hummel et al. 2013) and MIRC (Schaefer 2013). From these interferometric data, Hummel et al. (2013) and Schaefer (2013) independently proposed a distance $d \sim 385 \mathrm{pc}$. Although optical interferometry will soon provide uncertainties of 1\%-2\% for the distance to $\sigma$ Ori (G. Schaefer et al., in preparation), we assumed a more conservative error of 5\%, which translates into a heliocentric distance of $d=385 \pm 19$ pc.

\subsection{The Elusive $\sigma$ Ori B Component}

Bolton (1974) was one of the first authors to postulate the presence of a third component in the $\sigma$ Ori A, B system (although Frost \& Adams 1904, had done it seven decades earlier). He commented that the velocities and "spectroscopic" magnitude difference shown in one of his spectrograms indicated that none of the spectroscopic components were the component B of the visual system and, furthermore, that there was no evidence of $\mathrm{B}$ in his spectrograms. As shown below, our present-day, betterquality, observational data set confirms Bolton's statements.

Following the estimations by Hartkopf et al. (1996), component B should not be separated by more than $\sim 3 \mathrm{~km} \mathrm{~s}^{-1}$ from the systemic velocity of the $\mathrm{Aa}, \mathrm{Ab}$ pair. From the difference in magnitude relative to the $\mathrm{Aa}, \mathrm{Ab}$ component, the $\mathrm{B}$ star should have a spectral type $\sim \mathrm{B} 0-\mathrm{B} 2 \mathrm{~V}$. Thanks to the large radial velocities reached by $\sigma$ Ori Aa and Ab during periastron passage (separated by up to $292 \mathrm{~km} \mathrm{~s}^{-1}$; see Table 1), it might be possible to find spectroscopic features of the B component around phase zero. However, we did not find any sign of lines from an early B star close to the systemic velocity in any of the spectra obtained during periastron passage. This fact is illustrated by Figure 5, where we show the spectrum of $\sigma$ Ori $\mathrm{Aa}, \mathrm{Ab}, \mathrm{B}$ with maximum separation between the lines of the $\mathrm{Aa}$ and $\mathrm{Ab}$ components together with high-S/N spectra of two well-investigated early B dwarfs. The only possibility to reconcile photometry (i.e., the celebrated astrometric "binary" announced by Burnham in 1892) and spectroscopy (i.e., the absence of lines at $V_{r} \approx \gamma$ ) is to consider that $\sigma$ Ori $\mathrm{B}$ has a large rotational velocity, of $v \sin i \sim 200-300 \mathrm{~km} \mathrm{~s}^{-1}$.

\subsection{Stellar Parameters of $\sigma$ Ori $A a, A b$, and $B$}

\subsubsection{Spectroscopic Parameters}

We used the HERMES spectrum taken on 2013 October 11, the one with the largest separation between the lines of the $\mathrm{Aa}$ and $\mathrm{Ab}$ components, to perform a combined quantitative spectroscopic analysis of the $\sigma$ Ori Aa, Ab, B system. By

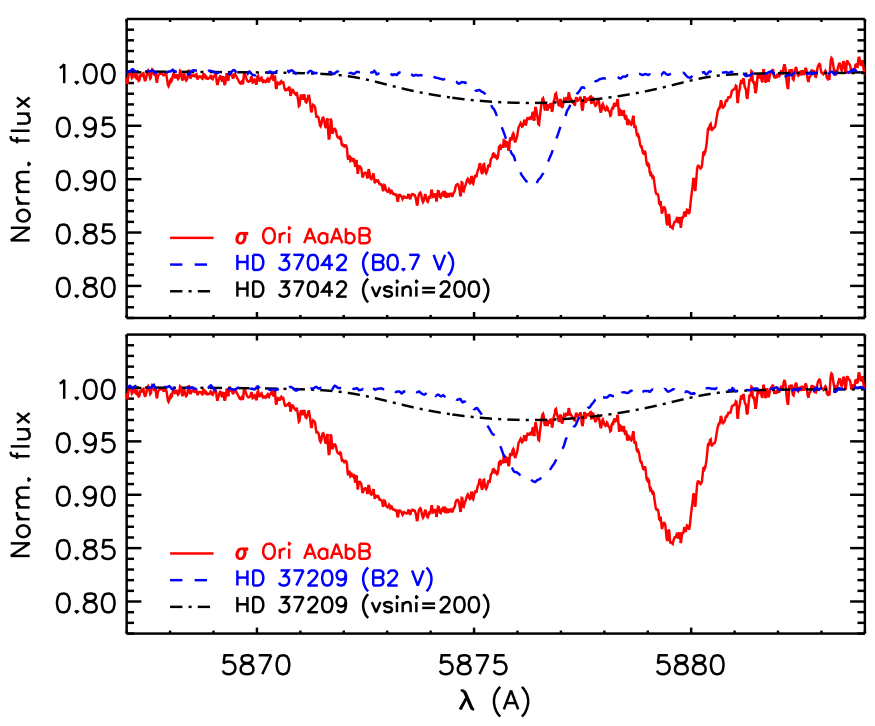

Figure 5. He I $\lambda 5875$ line in the HERMES spectra of HD 37042 (B0.7V, upper panel) and HD 37209 (B2V, lower panel) overplotted on the HERMES spectrum of $\sigma$ Ori Aa, Ab, B obtained on 2013 November 10 (red solid line). The spectra of the comparison stars have been shifted to the position where $\sigma$ Ori B should be found $\left(V_{r}=\gamma=31.10 \mathrm{~km} \mathrm{~s}^{-1}\right)$ and diluted to account for the difference in magnitude between the $\mathrm{Aa}, \mathrm{Ab}$, and $\mathrm{B}$ components. Black dot-dashed lines: spectra of the comparison stars convolved to $v \sin i=200 \mathrm{~km} \mathrm{~s}^{-1}$; blue dashed lines: original diluted spectra.

combined we mean that the stellar parameters of the three components were obtained directly and simultaneously from the analysis of one of the original spectra. This type of analysis can be considered as opposite to the spectral disentangling option, in which the spectrum of each component is obtained and analyzed separately. The combined synthetic spectra to be fitted to the observed one were constructed using spectra from the grid of FASTWIND models with solar metallicity (González Hernández et al. 2008; Simón-Díaz 2010; Nieva \& Simón-Díaz 2011) included in IACOB-GBAT (Simón-Díaz et al. 2011c). The spectra of each component were convolved to the corresponding $v \sin i$, shifted in radial velocity, and scaled by a certain factor $d_{i}$, where $\sum d_{i}=1$. Then, the three synthetic spectra were added together and the combined spectrum overplotted to the observed one.

We fixed in our analysis the radial and projected rotational velocities of the three components. For components Aa and $\mathrm{Ab}$ we considered the values directly measured from the 2013 October 11 spectrum itself (see Table 4), while for component B we initially assumed a $v \sin i=200 \mathrm{~km} \mathrm{~s}^{-1}$ and a radial velocity equal to the systemic velocity of the Aa, Ab system (note the difference between the inclination $i$ of the rotation axis of each component, as quoted in $v \sin i$, and the inclination of the orbit of the Aa, Ab system, as quoted in $M_{\text {dyn }} \sin ^{3} i$ in Table 1). In view of the results from the spectroscopic analysis by Najarro et al. (2011), we also fixed the associated helium abundances $\left(Y_{\mathrm{He}}=0.10\right)$, microturbulence velocities $\left(\xi_{\mathrm{t}}=5 \mathrm{~km} \mathrm{~s}^{-1}\right)$, and wind-strength parameters $(\log Q=-14.0)$ for the three stars. Last, we made use of the magnitude differences of previous sections to estimate the dilution factors. In particular, the Aa, $\mathrm{Ab}$, and $\mathrm{B}$ components contributed to the global spectrum by $48 \%, 28 \%$, and $24 \%$, respectively. As a result, only the effective temperatures $\left(T_{\text {eff }}\right)$ and gravities $(\log g)$ remained as free parameters to be determined in the analysis.

The best-fit solution was obtained by visual comparison of the original and combined synthetic spectra around the $\mathrm{H}$ and 

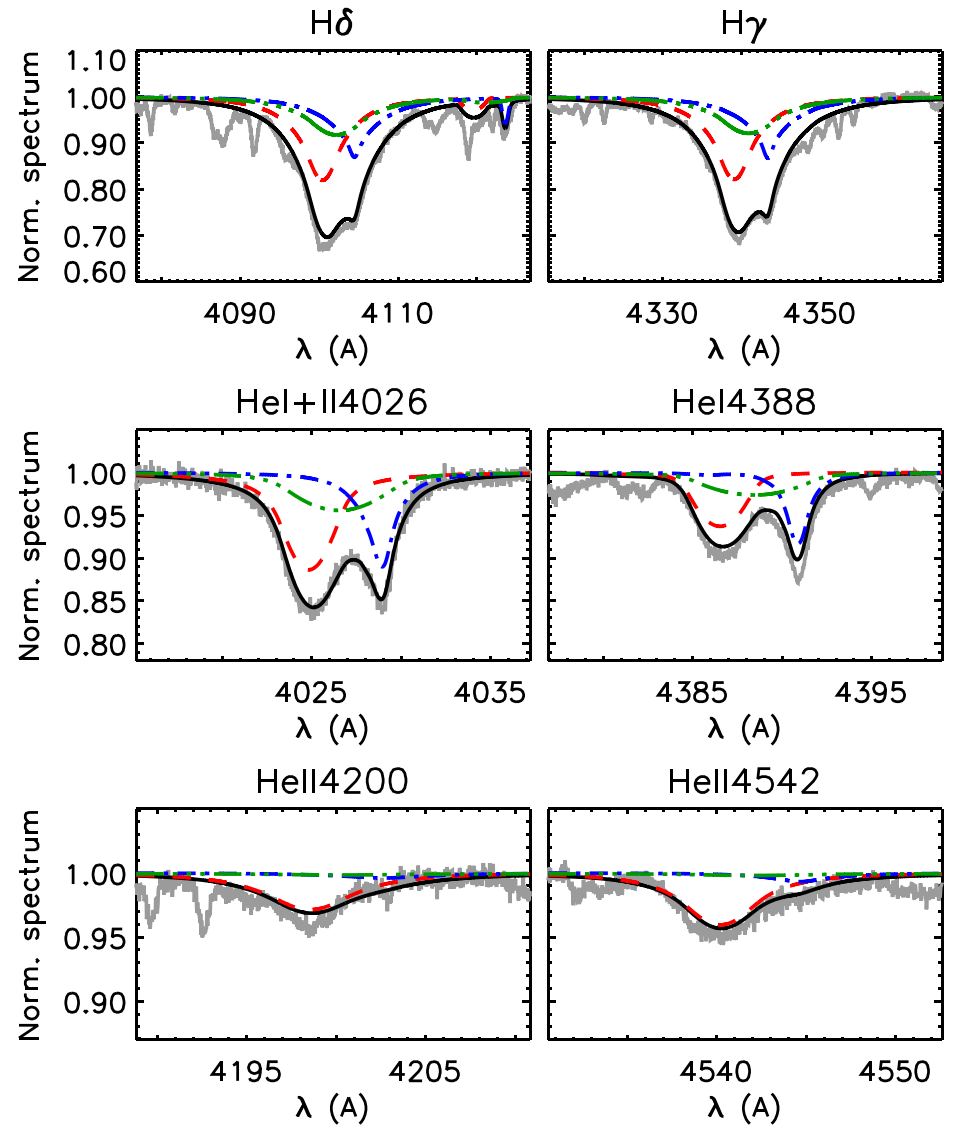

Hel4388

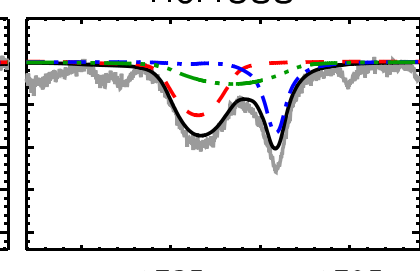

4385


Hel4471
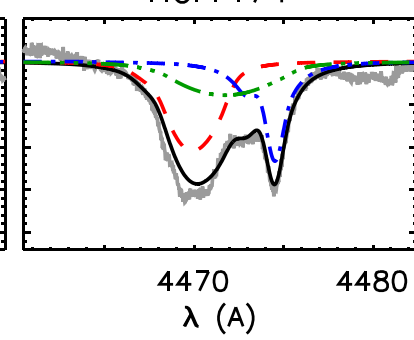

4480
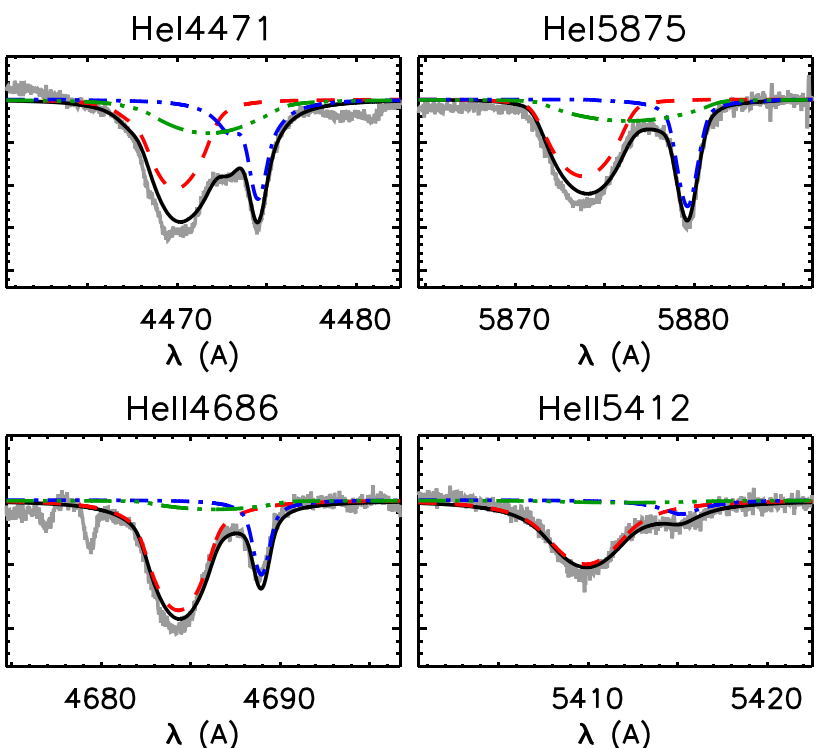

$\lambda(A)$

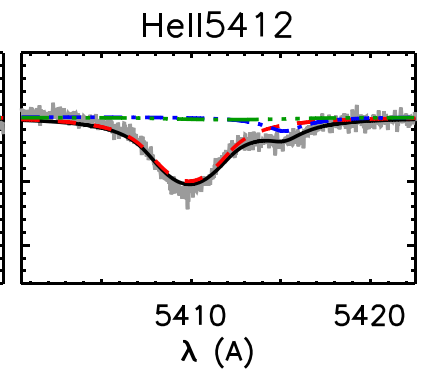

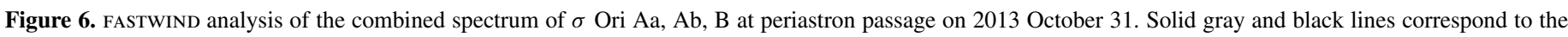

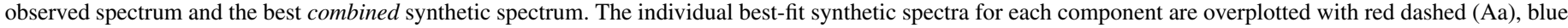
dash-dotted (Ab), and green long-dash-dotted (B) lines.

Table 4

Stellar Properties of $\sigma$ Ori Aa, Ab, and B

\begin{tabular}{lcccc}
\hline \hline Parameter & $\sigma$ Ori Aa & $\sigma$ Ori Ab & $\sigma$ Ori B & Unit \\
\hline$V$ & $4.61 \pm 0.02$ & $5.20 \pm 0.03$ & $5.31 \pm 0.04$ & $\mathrm{mag}$ \\
$M_{V}$ & $-3.49 \pm 0.11$ & $-2.90 \pm 0.11$ & $-2.79 \pm 0.12$ & $\mathrm{mag}$ \\
$v \sin i$ & $135 \pm 15$ & $35 \pm 5$ & $250 \pm 50$ & $\mathrm{~km} \mathrm{~s}^{-1}$ \\
$T_{\text {eff }}$ & $35.0 \pm 1.0$ & $31.0 \pm 1.0$ & $29.0 \pm 2.0$ & $\mathrm{kK}^{-2}$ \\
$\log g$ & $4.20 \pm 0.15$ & $4.20 \pm 0.15$ & $4.15 \pm 0.20$ & $\mathrm{~cm} \mathrm{~s}^{-2}$ \\
$\log g_{\mathrm{c}}$ & $4.21 \pm 0.15$ & $4.20 \pm 0.15$ & $4.18 \pm 0.20$ & $\mathrm{~cm} \mathrm{~s}^{-2}$ \\
$R$ & $5.6 \pm 0.3$ & $4.8 \pm 0.3$ & $5.0 \pm 0.3$ & $R_{\odot}$ \\
$\log L / L_{\odot}$ & $4.62 \pm 0.07$ & $4.27 \pm 0.07$ & $4.20 \pm 0.13$ & \\
$M_{\mathrm{sp}}$ & $18 \pm 7$ & $13 \pm 5$ & $14 \pm 7$ & $M_{\odot}$ \\
$M_{\mathrm{ev}}{ }^{\mathrm{a}}$ & $20.0_{-1.0}^{+0.9}$ & $14.6_{-0.6}^{+0.8}$ & $13.6_{-0.9}^{+1.1}$ & $M_{\odot}$ \\
Age & $0.3_{-0.3}^{+1.0}$ & $0.9_{-0.9}^{+1.5}$ & $1.9_{-1.9}^{+1.6}$ & $\mathrm{Ma}^{\mathrm{b}}$ \\
$v_{\mathrm{rot}, \text { ini }}$ & $150_{-50}^{+60}$ & $40_{-35}^{+43}$ & $270_{-70}^{+86}$ & $\mathrm{~km} \mathrm{~s}^{-1}$ \\
$\log Q\left(\mathrm{H}^{0}\right)$ & 47.92 & 47.03 & 46.34 & $\mathrm{~s}^{-1}$ \\
$\log Q\left(\mathrm{He}^{0}\right)$ & 46.25 & 44.58 & 43.35 & $\mathrm{~s}^{-1}$ \\
\hline
\end{tabular}

Notes.

${ }^{a} M_{\text {ev }}$ refers to present-day evolutionary masses; however, owing to the youth and low mass-loss rates of the stars, present-day and initial masses are equal.

$\mathrm{b}$ The quoted uncertainties in the estimated ages may actually be considered as upper limits since BONNSAI computations performed for this study assume that there is no correlation between the uncertainties associated with $T_{\text {eff }}, \log L$, and $\log g$ (see notes in Section 3.4.2 and Figure 7).

He I-II lines, which are commonly assumed as a diagnosis for the determination of stellar parameters of $\mathrm{O}$ stars (e.g., Herrero et al. 1992, 2002; Repolust et al. 2004). The fit is illustrated by Figure 6, which also shows the relative contribution of each component to the global spectrum. Although the contribution of the $\mathrm{B}$ component to the combined $\mathrm{H}$ and $\mathrm{He} \mathrm{I}$ lines is very small, the presence of a third component is needed to fit the region between the lines of the $\mathrm{Aa}$ and $\mathrm{Ab}$ components. On the other hand, the contribution of $\mathrm{B}$ to the He II lines is negligible, as expected from its lower effective temperature and large $v \sin i$.

Figure 6 also illustrates the difficulty to determine precisely the gravity of the three components (from the wings of the hydrogen Balmer lines) and the effective temperature of the elusive B component (mainly constrained by the weak He II lines). To improve this situation, we followed an iterative strategy that accounted for the comparison of spectroscopic and evolutionary masses (Section 3.4.2). The resulting spectroscopic parameters are summarized in Table 4. The derived $T_{\text {eff }}$ and $\log g$ are also complemented with the gravities corrected from centrifugal acceleration ( $\log g_{\mathrm{c}}$, computed following the procedure described in Repolust et al. 2004).

\subsubsection{Physical Parameters}

Once effective temperatures and gravities were determined, we used them, along with the spectral energy distributions of the associated FASTWIND models and the absolute visual magnitudes computed in Section 3.2, to obtain the radii, luminosities, and spectroscopic masses $\left(M_{\mathrm{sp}}\right)$ of the three components. To this aim, we followed the same strategy as described in Herrero et al. (1992). The resulting stellar parameters and associated uncertainties from FASTWIND are given in Table 4. The errors in $R, \log L$, and $M_{\text {sp }}$ take into account the uncertainties in distance, extinction, effective temperatures, gravities, and individual extinction-corrected $V$-band absolute magnitudes (obtained 
in turn from the uncertainties in the combined apparent magnitude and magnitude differences between $\mathrm{A}$ and $\mathrm{B}$ and between $\mathrm{Aa}$ and $\mathrm{Ab}$; see Section 3.2).

We then used the Bayesian code BONNSAI ${ }^{15}$ (Schneider et al. $2014 \mathrm{~b})$ to infer the evolutionary masses $\left(M_{\mathrm{ev}}\right)$ and ages of $\sigma$ Ori $\mathrm{Aa}, \mathrm{Ab}$, and $\mathrm{B}$. Once some priors are accounted for, BONNSAI matches all available observables simultaneously to stellar models and delivers probability distributions of the derived stellar parameters. In our case, we matched the luminosities, surface gravities, effective temperatures, and projected rotational velocities of our three stars to the rotating Milky Way stellar models of Brott et al. (2011). We assumed a Salpeter initial mass function (Salpeter 1955) as initial mass prior, uniform priors for age and initial rotational velocity, and that all rotation axes are randomly distributed in space. The stellar models reproduced correctly the observables. The determined initial masses, ages, and initial rotational velocities are summarized in Table 4 including their $1 \sigma$ uncertainties.

For completeness, we also provide in Table 4 the number of hydrogen $\left(\mathrm{H}^{0}\right)$ and neutral helium $\left(\mathrm{He}^{0}\right)$ ionizing photons emitted by $\sigma$ Ori Aa, Ab, and B. These quantities can be of future interest for, e.g., the study of the ionization of the Horsehead Nebula, the associated photodissociation region, and the impact of massive stars on the formation and evolution of low-mass stars, brown dwarfs, stellar disks, Herbig-Haro objects, and possible planetary systems in the $\sigma$ Orionis star-forming region. In total, the triple system emits $9.6 \times 10^{47} \mathrm{H}^{0}$ and $1.8 \times 10^{46} \mathrm{~s}^{-1}$ $\mathrm{He}^{0}$ ionizing photons, enough for shaping virtually all remnant clouds in the Ori OB1b association (Ogura \& Sugitani 1998). However, the ionization budget of the surrounding interstellar medium is mainly dominated by the hottest component only, $\sigma$ Ori Aa, which contributes with $87 \%\left(\mathrm{H}^{0}\right)$ and $98 \%\left(\mathrm{He}^{0}\right)$ of the total numbers of ionizing photons.

\section{DISCUSSION}

\subsection{Summary of Orbital Properties of the $\sigma$ Ori $A a, A b, B$ System}

As shown before, the angular separation between $\sigma$ Ori Aa, $\mathrm{Ab}$ and $\mathrm{B}$ lies today at $\sim 0.25$, which translates into a projected physical separation of about $100 \mathrm{AU}$ (Caballero 2014, and references therein). This separation makes the "pair" resolvable only with the Hubble Space Telescope and, from the ground, with micrometers, speckle, adaptive optics, or lucky imagers at $>1$ m class telescopes.

The last published orbital solution, by Turner et al. (2008), quoted a period $P=156.7 \pm 3.0$ a, an eccentricity $e=0.0515 \pm$ 0.0080 , and an inclination angle $i=159.7 \pm 3.7 \mathrm{deg}$ (i.e., the orbit is almost circular and located in the plane of the sky). Our AstraLux astrometric measurements (Table 2), together with many other independent ones obtained after 2008, will certainly help to refine the published orbital solution.

Regarding the $\sigma$ Ori Aa, Ab pair, over a century had to elapse since the first suspicion of spectroscopic binarity by Frost \& Adams (1904), through the first quantitative measures by Bolton (1974) and Stickland \& Lloyd (2001), to the confirmation by Simón-Díaz et al. (2011a). With a projected physical separation between 0.8 and 6.4 mas, the $\mathrm{Aa}$, Ab system can be resolved in imaging only with interferometric observations. After publication of Simón-Díaz et al. (2011a), and during the development of this paper, two different teams have been able to resolve the

\footnotetext{
15 http://www.astro.uni-bonn.de/stars/bonnsai
}

$\mathrm{Aa}, \mathrm{Ab}$ pair with interferometric facilities (Hummel et al. 2013; Schaefer 2013). While they plan to publish a joint analysis (D. Gies, 2014 private communication), both groups have already indicated $57 \mathrm{deg}$ and $56.3 \mathrm{deg}$, respectively, as first estimates for the inclination orbit of the $\mathrm{Aa}, \mathrm{Ab}$ system. Our study allows us to provide an independent rough estimation of the inclination of the $\mathrm{Aa}, \mathrm{Ab}$ orbit based on the comparison of our own determinations of the projected dynamical masses and evolutionary masses (see Sections 3 and 4.3). Considering the values of $M_{\text {dyn }} \sin ^{3} i$ and $M_{\mathrm{ev}}$ for the $\mathrm{Aa}$ and $\mathrm{Ab}$ components indicated in Table 4, we obtained $i=52.0 \pm 1.2 \mathrm{deg}$ and $52.9 \pm 1.2 \mathrm{deg}$, respectively. These values are in relatively good agreement with (but a bit smaller than) the preliminary estimations provided by the combination of spectroscopic and interferometric observations.

All the quoted values of $i$ imply that the $\sigma$ Ori Aa, Ab system is not eclipsing. We have confirmed this result using Hipparcos photometry: we did not find any indication of possible eclipses, which should occur at phases 0.02 and 0.95 and by folding the light curve to periods close to 143 days.

In light of the orbital properties and derived projected rotational velocities of $\sigma$ Ori Aa, Ab, B (Table 4), we conclude that the geometry of this young hierarchical triple system is rather complex. On one hand, the close pair revolves in a very eccentric orbit with an inclination $\sim 55 \mathrm{deg}$, while the wide pair does it in an almost circular orbit and with an inclination of $\sim 160 \mathrm{deg}$. Therefore, the triple system is far from being coplanar. On the other hand, the projected rotational velocities of the Aa and Ab components are very different (135 and $35 \mathrm{~km} \mathrm{~s}^{-1}$, respectively). In absence of direct information about the inclination angle of the rotational axes, we hypothesize that the spins of the two stars are not synchronized and/or the rotational axes are not aligned. A more thorough investigation of the global properties of $\sigma$ Ori Aa, Ab, B in the context of the statistical properties, formation, and evolution of multiple stellar systems, as in Tokovinin (2008), is one possible direction of future work.

The good phase coverage of our spectroscopic observations and the great accuracy in the determination of the orbital period of the very eccentric binary $\sigma$ Ori $\mathrm{Aa}, \mathrm{Ab}$ (with an error of 7.2 minutes in 143.2 days) allowed us to identify precisely the future dates of periastron and apoastron passages until 2020. The passage dates shown in Table 5 will certainly ease the investigation of, e.g., apsidal motion, circularization, orbital period variation, and, especially, X-ray emission (see below).

\section{2. $\sigma$ Ori Aa, Ab, B as an X-Ray Emitter}

For years, $\sigma$ Ori Aa, Ab, B has been identified as the most luminous and softest X-ray source in the $\sigma$ Orionis cluster (Berghoefer \& Schmitt 1994; Sanz-Forcada et al. 2004; Skinner et al. 2008; Caballero et al. 2009, 2010). From a comprehensive analysis of high-resolution Chandra X-ray imaging and spectroscopy of the triple system (none of the Aa, Ab, B components can be resolved by existing X-ray telescopes), Skinner et al. (2008) concluded that the measured X-ray properties of $\sigma$ Ori were in good agreement with model predictions for shocks distributed in a radiatively driven stellar wind. However, other possible emission mechanisms that could not be ruled out by that time were a magnetically confined wind shock in the weak-field limit and a subterminal speed-colliding wind system, under the hypothesis that $\sigma$ Ori A had a putative spectroscopic companion, which had not been confirmed yet. With our latest results, the estimated minimum and maximum separations between the $\mathrm{Aa}$ and $\mathrm{Ab}$ components are $\sim 66$ and $\sim 528 R_{\odot}$ (about 10 and 
Table 5

Next Epochs of Quadratures of the $\sigma$ Ori Aa, Ab Pair

\begin{tabular}{|c|c|c|c|}
\hline \multicolumn{2}{|c|}{ Periastron } & \multicolumn{2}{|c|}{ Apastron } \\
\hline $\begin{array}{l}\text { Date } \\
\text { (yyyy mm dd) }\end{array}$ & $\begin{array}{c}\text { HJD } \\
(-2,450,000)\end{array}$ & $\begin{array}{c}\text { Date } \\
\text { (yyyy mm dd) }\end{array}$ & $\begin{array}{c}\text { HJD } \\
(-2,450,000)\end{array}$ \\
\hline 2015 Jan 4 & $7027.22 \pm 0.04$ & 2015 Mar 17 & $7098.82 \pm 0.04$ \\
\hline 2015 May $27^{*}$ & $7170.42 \pm 0.04$ & 2015 Aug $7^{*}$ & $7242.01 \pm 0.04$ \\
\hline 2015 Oct 18 & $7313.61 \pm 0.05$ & 2015 Dec 28 & $7385.21 \pm 0.05$ \\
\hline 2016 Mar 9 & $7456.81 \pm 0.05$ & 2016 May $19 *$ & $7528.41 \pm 0.05$ \\
\hline 2016 Jul 30* & $7600.01 \pm 0.06$ & 2016 Oct 10 & $7671.61 \pm 0.06$ \\
\hline 2016 Dec 20 & $7743.21 \pm 0.06$ & 2017 Mar 2 & $7814.81 \pm 0.06$ \\
\hline 2017 May 12* & $7886.40 \pm 0.07$ & 2017 Jul 23* & $7958.00 \pm 0.07$ \\
\hline 2017 Oct 3 & $8029.60 \pm 0.07$ & 2017 Dec 13 & $8101.20 \pm 0.07$ \\
\hline 2018 Feb 23 & $8172.80 \pm 0.08$ & 2018 May $5^{*}$ & $8244.40 \pm 0.08$ \\
\hline 2018 Jul 16* & $8316.00 \pm 0.08$ & 2018 Sep 26 & $8387.600 \pm 0.08$ \\
\hline 2018 Dec 6 & $8459.20 \pm 0.09$ & 2019 Feb 16 & $8530.80 \pm 0.09$ \\
\hline 2019 Apr $28^{*}$ & $8602.40 \pm 0.09$ & $2019 \mathrm{Jul} 9 *$ & $8673.99 \pm 0.09$ \\
\hline 2019 Sep 19 & $8745.59 \pm 0.10$ & 2019 Nov 29 & $8817.19 \pm 0.10$ \\
\hline 2020 Feb 9 & $8888.79 \pm 0.10$ & 2020 Apr $20^{*}$ & $8960.39 \pm 0.10$ \\
\hline $2020 \mathrm{Jul} 1^{*}$ & $9031.99 \pm 0.11$ & 2020 Sep 11 & $9103.59 \pm 0.11$ \\
\hline 2020 Nov 21 & $9175.19 \pm 0.11$ & 2012 Feb 1 & $9246.79 \pm 0.11$ \\
\hline
\end{tabular}

Notes. Dates marked with an asterisk denote that $\sigma$ Ori is not observable as a result of its proximity to the Sun.

100 stellar radii), respectively. Although previous investigations of X-ray emission variability have resulted in negative evidence (Sanz-Forcada et al. 2004; Skinner et al. 2008; Caballero et al. 2010), likely owing to the relatively long period of 143.2 days and high eccentricity, the colliding wind shock hypothesis, now the most probable scenario, can be tested during future periastron passages with either Chandra or XMM-Newton.

\subsection{Stellar Masses}

We provided a rigorous determination of the evolutionary masses and ages of the three components with the BONNSAI tool in Section 3.4.1. Complementing these estimates, the Hertzsprung-Russell diagram in Figure 7 allows a quick visual estimation of their evolutionary masses and ages, their associated uncertainties, and how these quantities are modified when the estimated effective temperatures and assumed distance are modified. We derived a total evolutionary mass $M_{\mathrm{Aa}+\mathrm{Ab}+\mathrm{B}}=48.2$ $\pm 1.5 M_{\odot}$, a value that is in good agreement with the total $d y$ namical mass derived by means of Kepler's third law assuming the Turner et al. (2008) orbital parameters for the "astrometric" pair and a distance of $385 \mathrm{pc}\left(M_{\mathrm{A}+\mathrm{B}}=44 \pm 7 M_{\odot}\right)$. We also found fair matches between the ratios of evolutionary masses $(1.37 \pm 0.10)$ and those of dynamical masses $(1.325 \pm 0.006$; Table 1) of the $\mathrm{Aa}$ and $\mathrm{Ab}$ components. Finally, our derived evolutionary masses for the inner pair are systematically larger than the values resulting from the analysis of the interferometric observations $\left(M_{\text {dyn }, \mathrm{Aa}}=16.7\right.$ and $M_{\text {dyn, } \mathrm{Ab}}=12.6 M_{\odot}$; Hummel et al. 2013; G. Schaefer, 2014 private communication). This is a puzzling result since the effective temperatures that would lead to these evolutionary masses ( $\sim 32$ and $29 \mathrm{kK}$, respectively; see Figure 7) are incompatible with the values indicated by the quantitative spectroscopic analysis (see also notes in Section 4.4). A possible solution to this relative mismatch between the evolutionary and dynamical masses could be related to the inclination angle resulting from the combined radial velocity and interferometric analysis. In particular, taking into account the preliminary dynamical masses of $\sigma$ Ori Aa and $\sigma$ Ori $\mathrm{Ab}$ computed by Hummel et al. (2013) and Schaefer (2013) and the

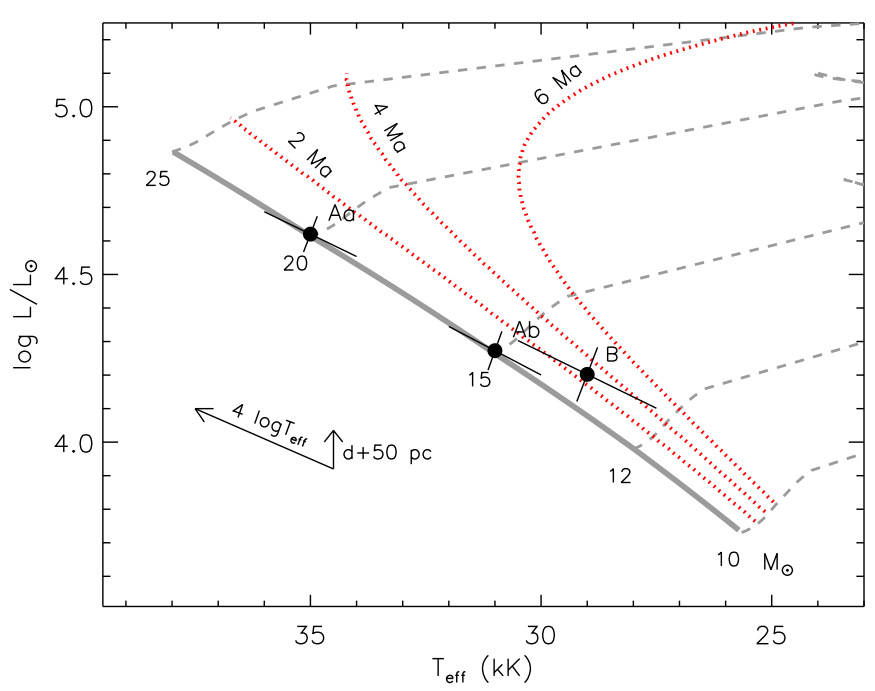

Figure 7. Hertzsprung-Russel diagram showing the three components of $\sigma$ Ori $\mathrm{Aa}, \mathrm{Ab}, \mathrm{B}$, and evolutionary tracks (gray dashed), ZAMS line (black solid), and 2, 4, and $6 \mathrm{Ma}$ isochrones for stellar masses in the range 10-25 $M_{\odot}$ and solar metallicity from Brott et al. (2011) models. Sizes of error bars of effective temperature and luminosity of $\sigma$ Ori $\mathrm{Aa}, \mathrm{Ab}$, and $\mathrm{B}$ were computed as if both quantities were not correlated. The diagonal and vertical arrows indicate the expected direction of correlated variability of $T_{\mathrm{eff}}$ and $L$, and of $L$ when heliocentric distance is increased by $50 \mathrm{pc}$, respectively.

total $\mathrm{Aa}+\mathrm{Ab}+\mathrm{B}$ mass indicated above $\left(44 \pm 7 M_{\odot}\right)$, the B component would be more massive than the Ab component, while the latter is more luminous. Using our evolutionary masses, there is a better correspondence between mass and luminosity regarding the three more massive components of $\sigma$ Ori. As indicated in Section 4.1, an orbit with a slightly smaller inclination would solve this mismatch between dynamical masses and luminosity.

\subsection{Stellar Ages}

The age of the $\sigma$ Orionis cluster has been extensively investigated in the literature, mainly using low-mass stars (e.g., Zapatero Osorio et al. 2002; Sherry et al. 2004, 2008; Caballero 2007; Mayne \& Naylor 2008). The widely accepted cluster age interval is $2-4 \mathrm{Ma}$, with extreme values reported at 1.5 and $8 \mathrm{Ma}$. Our study provides an independent determination of the age of the three most massive stars in the cluster, namely, $\sigma$ Ori $\mathrm{Aa}, \mathrm{Ab}$, and $\mathrm{B}$. The individual stellar ages indicated by BONNSAI are $0.3_{-0.3}^{+1.0}, 0.9_{-0.9}^{+1.5}$, and $1.9_{-1.9}^{+1.6} \mathrm{Ma}$, respectively. In view of these values, one could argue that the derived ages for the three components are in agreement (within the uncertainties) and propose a mean age of $\sim 1 \mathrm{Ma}$ for the $\sigma$ Ori Aa, Ab, B system. This result confirms the youth of the $\sigma$ Orionis cluster but also points toward stellar ages of the most massive members (especially $\sigma$ Ori Aa and $\sigma$ Ori $\mathrm{Ab}$ ), which are slightly younger than the commonly accepted cluster age. The latter statement is reinforced when one takes into account that the quoted uncertainties in the ages resulting from the BONNSAI analysis may be actually considered as upper limits. The BONNSAI computations assume that the uncertainties in the three input parameters $\left(T_{\text {eff }}, \log L\right.$, and $\log g$ ) are independent; however, as illustrated in Figure 7, there is a strong correlation (linked to basic principles of stellar astrophysics) between variations in $\log L$ and $T_{\text {eff }}$. These variations follow an inclined line in the H-R diagram almost parallel to the zero-age main sequence (ZAMS) and the 1-4 Ma isochrones in the $10-20 M_{\odot}$ range. As a consequence, the uncertainty in the derived age due to uncertainties related to the 
effective temperature determination are actually smaller than predicted by BONNSAI.

Being aware of the subtlety of the result about the difference between the age derived for $\sigma$ Ori $\mathrm{Aa}, \mathrm{Ab}(\leqslant 1 \mathrm{Ma})$ and the commonly accepted cluster age (2-4 Ma), we also wanted to investigate further whether this non-coevality is a result of an underestimation of the uncertainties in the derived ages or could be used as an observational evidence of an actual physical process linked to the formation or evolution of these stars.

We first discarded it to be a consequence of an incorrect determination of the stellar parameters (especially effective temperatures), cluster distance, or individual magnitudes. Any of these three possibilities can locate $\sigma$ Ori Aa and $\sigma$ Ori Ab in a reasonable way on the $2 \mathrm{Ma}$ isochrone. However, an effective temperature lower than $32 \mathrm{kK}$ for $\sigma$ Ori Aa would deliver a bad fitting of the He I-II ionization equilibrium (the same argument applies, to a less extent, to $\sigma$ Ori $\mathrm{Ab}$ ); a further distance, of $430 \mathrm{pc}$, would translate into an age of about $6 \mathrm{Ma}$ for $\sigma$ Ori $\mathrm{B}$; and the magnitude difference between $\sigma$ Ori $\mathrm{Aa}, \mathrm{Ab}$ and $\mathrm{B}$ necessary to put the three components simultaneously on the ZAMS is incompatible with observations ( $\Delta V \sim 1.5 \mathrm{mag}$ ).

The assumed metallicity and input physics considered in the stellar evolution models could also modify the derived ages. To check the effect of these parameters, we compared the location of the tracks and isochrones computed by the Bonn (Brott et al. 2011) and Geneva (Ekström et al. 2012) groups, which assumed a slightly different solar metallicity and coreovershooting parameter. The position of the ZAMS (for the same initial rotational velocity) in Ekström et al. (2012) models is exactly the same as for Brott et al. (2011); on the other hand, the isochrones of a given age in Ekström et al. (2012) computations are closer to the ZAMS compared to Brott et al. (2011) models. As a consequence, Geneva models implied slightly older ages for $\sigma$ Ori Aa and $\sigma$ Ori $\mathrm{Ab}$; however, these were still less than $1 \mathrm{Ma}$.

We also explored the possibility that $\sigma$ Ori Aa and $\sigma$ Ori Ab have suffered from a rejuvenation process by mass accretion (viz., Braun \& Langer 1995; van Bever \& Vanbeveren 1998; Dray \& Tout 2007; Schneider et al. 2014a). As indicated in Section 4.1 , these two stars with masses $\sim 20$ and $\sim 15 M_{\odot}$ are orbiting in a high eccentric orbit with a period of 143.2 days. The closest separation between the stars in the orbit, at periastron, is $\sim 65 R_{\odot}$ (more than a factor of 10 in stellar radii). This wide separation, together with the small tabulated mass-loss rate (Najarro et al. 2011), makes the occurrence of mass transfer episodes (and hence any associated rejuvenation phenomenon) in this system very unlikely.

Are we then facing empirical evidence of a star formation process in which massive stars in a stellar cluster are formed slightly after their low-massive counterparts? The formation of massive stars is still an open, highly debated question. Zinnecker $\&$ Yorke (2007) reviewed the three main competing concepts of massive star formation, including (1) the monolithic collapse in isolated cores, (2) the competitive accretion in a protocluster environment, and (3) stellar collisions and mergers in very dense clusters. In absence of any other satisfactory solution at this point, we might assume the competitive accretion scenario as a likely explanation for the slightly younger age derived for $\sigma$ Ori $\mathrm{Aa}, \mathrm{Ab}$ compared to cluster age as determined from lowmass stars. Although it must be considered for the moment as a highly speculative statement, its confirmation/refutation (based on a much deeper study including all possible observational information regarding the high- and low-mass content of the $\sigma$
Orionis cluster and its viability in terms of spatial and temporal scales) deserves further investigation. In this context, we note the recent study by Rivilla et al. (2013), in which the authors claim that the scenario that better explains the distribution of premain-sequence stars in three star formation regions in Orion (the Trapezium cluster, the Orion hot core, and the OMC1$\mathrm{S}$ region) assumes high fragmentation in the parental core, accretion at subcore scales that forms a low-mass stellar cluster, and subsequent competitive accretion.

\section{SUMMARY}

The almost extinction-free, young $\sigma$ Orionis cluster in the Ori $\mathrm{OB} 1 \mathrm{~b}$ association is a cornerstone region for the study of stellar and substellar formation and the interplay between the strong (far-)UV radiation emitted by massive stars and the surrounding interstellar material. At the very center of the cluster, the massive $\sigma$ Ori A, B system, commonly identified as a close astrometric binary, has been recently confirmed to be a hierarchical triple system. The first two stars ( $\sigma$ Ori Aa and Ab) are coupled in a very eccentric orbit with a period $\sim 143$ days, and both together are orbiting with the third component ( $\sigma$ Ori B) in a much wider and longer $(P \sim 156.7$ a) orbit, almost circular.

Aiming at providing a complete characterization of the physical properties of the three components and an improved set of orbital parameters for the highly eccentric $\sigma$ Ori Aa, Ab pair, we compiled and analyzed a spectroscopic data set comprising 90 high-resolution spectra $(\sim 33$ of them were obtained near periastron passage of the $\mathrm{Aa}-\mathrm{Ab}$ system). The complete sample covers a total time span of almost 14 orbital periods of the $\sigma$ Ori Aa, Ab system.

The revised orbital analysis of the radial velocity curves of the $\sigma$ Ori Aa, Ab pair led to an improved orbital solution compared to our previous study performed in Simón-Díaz et al. (2011a). The great accuracy reached in the determination of the orbital period (7.2 minutes in 143.198 days) allowed us to provide precise future ephemerides for the system. This can be of particular interest for the investigation of variability of the strong X-ray emission detected for $\sigma$ Ori A, B. In addition, the good phase coverage achieved by our observations settles a firm baseline for future investigations of apsidal motion effects, circularization, and/or time variation of the orbital period in this young, very eccentric massive stellar system.

We performed a combined quantitative spectroscopic analysis of the $\sigma$ Ori Aa, Ab, B system by means of the stellar atmosphere code FASTWIND. We used our own plus other available information about photometry and distance to the system to provide estimates for the radii, luminosities, and spectroscopic masses of the three components. We also inferred evolutionary masses and stellar ages using the Bayesian code BONNSAI.

Despite the absence of clear spectroscopic features associated with the $\sigma$ Ori B component in the combined spectrum, we provided indirect arguments indicating that the faintest star in the traditionally considered astrometric binary is an early B-type dwarf with a projected rotational velocity of at least $200 \mathrm{~km} \mathrm{~s}^{-1}$. The FASTWIND+BONNSAI analysis indicated that the $\sigma$ Ori Aa, Ab pair contains the hottest $\left(T_{\text {eff, } \mathrm{Aa}}=35.0 \pm 1.0\right.$ $\left.\mathrm{kK}, T_{\mathrm{eff}, \mathrm{Ab}}=31.0 \pm 1.0 \mathrm{kK}\right)$ and most massive $\left(M_{\mathrm{Aa}}=20.0 \pm\right.$ $\left.1.0 M_{\odot}, M_{\mathrm{Ab}}=14.6 \pm 0.7 M_{\odot}\right)$ components of the triple system, while $\sigma$ Ori B is a bit cooler and less massive $\left(T_{\text {eff, } \mathrm{B}}=29.0 \pm\right.$ $\left.1.5 \mathrm{kK}, M_{\mathrm{B}}=13.6 \pm 0.8 M_{\odot}\right)$. The derived stellar age for $\sigma$ Ori $\mathrm{B}\left(1.9_{-1.9}^{+1.6} \mathrm{Ma}\right)$ is in relatively good agreement with previous determinations of the age of the $\sigma$ Orionis cluster; however, the 
ages of the spectroscopic pair $\left(0.3_{-0.3}^{+1.0}\right.$ and $0.9_{-0.9}^{+1.5}$, respectively) are intriguingly younger than the commonly accepted cluster age (2-4 Ma).

The outcome of this study, once combined with ongoing interferometric and past/future optical and X-ray observations, will be of key importance for a precise determination of the distance to the $\sigma$ Orionis cluster, the interpretation of the strong X-ray emission of the $\sigma$ Ori Aa, Ab, B system, and the investigation of the formation and evolution of multiple massive stellar systems and substellar objects.

This research made use of SIMBAD, operated at Centre de Données astronomiques de Strasbourg, France, and NASA's Astrophysics Data System. Financial support was provided by the Spanish Ministerio de Ciencia e Innovación and Ministerio de Economía y Competitividad under grants AYA2010-17631, AYA2010-15081, AYA2010-21697-C05-04, AYA2011-30147C03-03, AYA2012-39364-C02-01/02, and Severo Ochoa SEV2011-0187, and by the Canary Islands Government under grant PID2010119. R.H.B. acknowledges support from FONDECYT Regular project 1140076 . J.S.-B. acknowledges support by the JAE-PreDoc program of the Spanish Consejo Superior de Investigaciones Científicas (CSIC). Á.S. acknowledges support by the János Bolyai Research Scholarship of the Hungarian Academy of Sciences. We acknowledge F. Najarro, J. Puls, A. Herrero, J. Casares, and H. Blau for interesting discussions during the development of this paper and their useful comments on one of the latest drafts before submission. We thank G. Schaefer for providing us some information about the results from the analysis of the interferometric observations prior to publication. Lastly, we thank the anonymous referee for a very pro- fessional report that helped us to improve the first version of the manuscript.

Based on observations made with the Nordic Optical Telescope, operated by the Nordic Optical Telescope Scientific Association at the Observatorio del Roque de los Muchachos, La Palma, Spain, of the Instituto de Astrofísica de Canarias. Based on observations made with the Mercator Telescope, operated on the island of La Palma by the Flemish Community, at the Spanish Observatorio del Roque de los Muchachos of the Instituto de Astrofisica de Canarias. Based on observations obtained with the HERMES spectrograph, which is supported by the Fund for Scientific Research of Flanders (FWO), Belgium, the Research Council of K.U. Leuven, Belgium, the Fonds National de la Recherche Scientifique (F.R.S.-FNRS), Belgium, the Royal Observatory of Belgium, the Observatoire de Genève, Switzerland, and the Thringer Landessternwarte Tautenburg, Germany. The Hobby-Eberly Telescope (HET) is a joint project of the University of Texas at Austin, the Pennsylvania State University, Stanford University, Ludwig-Maximilians-Universität München, and Georg-August-Universität Göttingen. The HET is named in honor of its principal benefactors, William P. Hobby and Robert E. Eberly.

Facilities: NOT, Mercator1.2m, CAO:2.2m, HET, Max Planck:2.2m

\section{APPENDIX \\ LOG OF OBSERVATIONS AND RADIAL VELOCITY MEASUREMENTS}

Table A1 shows the dates of the spectra obtained with the various spectrographs used in this paper, the associated measured radial velocities (see Section 3.1), and the corresponding phases following the orbital parameters indicated in Table 1.

Table A1

Radial Velocity Measurements of $\sigma$ Ori Aa and $\mathrm{Ab}$

\begin{tabular}{|c|c|c|c|c|c|c|c|}
\hline $\begin{array}{l}\text { Date } \\
\text { (yyyy mm dd) }\end{array}$ & $\begin{array}{c}\text { HJD } \\
(-2,450,000)\end{array}$ & $\phi$ & $\begin{array}{c}V_{r}(\mathrm{Aa}) \\
\left(\mathrm{km} \mathrm{s}^{-1}\right)\end{array}$ & $\begin{array}{c}O-C(\mathrm{Aa}) \\
\left(\mathrm{km} \mathrm{s}^{-1}\right)\end{array}$ & $\begin{array}{c}V_{r}(\mathrm{Ab}) \\
\left(\mathrm{km} \mathrm{s}^{-1}\right)\end{array}$ & $\begin{array}{c}O-C(\mathrm{Ab}) \\
\left(\mathrm{km} \mathrm{s}^{-1}\right)\end{array}$ & Instrument \\
\hline 2008 Nov 5 & 4776.735 & 0.284 & $50.1 \pm 3.5$ & -0.2 & $6.0 \pm 1.9$ & 0.3 & FIES \\
\hline 2008 Nov 5 & 4776.740 & 0.284 & $50.2 \pm 3.6$ & -0.1 & $7.0 \pm 1.9$ & 1.4 & FIES \\
\hline 2008 Nov 6 & 4777.697 & 0.291 & $50.4 \pm 3.6$ & 0.1 & $6.2 \pm 2.0$ & 0.5 & FIES \\
\hline 2008 Nov 6 & 4777.699 & 0.291 & $50.3 \pm 3.6$ & 0.0 & $6.3 \pm 2.0$ & 0.6 & FIES \\
\hline 2008 Nov 6 & 4777.700 & 0.291 & $50.4 \pm 3.5$ & 0.1 & $5.9 \pm 1.9$ & 0.2 & FIES \\
\hline 2008 Nov 7 & 4778.718 & 0.298 & $49.1 \pm 3.5$ & -1.1 & $8.0 \pm 1.9$ & 2.2 & FIES \\
\hline 2008 Nov 7 & 4778.719 & 0.298 & $49.3 \pm 3.5$ & -0.9 & $7.6 \pm 2.0$ & 1.8 & FIES \\
\hline 2008 Nov 8 & 4779.704 & 0.305 & $49.1 \pm 3.5$ & -1.0 & $6.3 \pm 2.0$ & 0.4 & FIES \\
\hline 2008 Nov 8 & 4779.705 & 0.305 & $46.9 \pm 3.6$ & -3.2 & $7.6 \pm 1.9$ & 1.7 & FIES \\
\hline 2009 May 2 & 4953.965 & 0.522 & $43.9 \pm 3.4$ & -1.5 & $12.0 \pm 1.9$ & -0.2 & FEROS \\
\hline 2009 May 3 & 4954.963 & 0.529 & $43.0 \pm 3.4$ & -2.2 & $12.8 \pm 2.0$ & 0.3 & FEROS \\
\hline 2009 Nov 9 & 5145.659 & 0.860 & $18.2 \pm 3.5$ & -0.5 & $48.0 \pm 1.9$ & 0.5 & FIES \\
\hline 2009 Nov 11 & 5147.650 & 0.874 & $14.4 \pm 3.4$ & -1.2 & $52.6 \pm 1.9$ & 0.9 & FIES \\
\hline 2010 Sep 7 & 5447.730 & 0.970 & $-45.8 \pm 1.3$ & 0.5 & $135.0 \pm 1.0$ & 1.3 & FIES \\
\hline 2010 Sep 9 & 5449.747 & 0.984 & $-74.2 \pm 1.5$ & 1.2 & $173.7 \pm 1.2$ & 1.5 & FIES \\
\hline 2010 Oct 21 & 5490.729 & 0.270 & $46.1 \pm 3.5$ & -4.3 & $8.6 \pm 1.9$ & 3.0 & HERMES \\
\hline 2010 Oct 23 & 5493.734 & 0.291 & $50.7 \pm 3.5$ & 0.4 & $6.2 \pm 2.0$ & 0.5 & FIES \\
\hline 2010 Oct 23 & 5493.736 & 0.291 & $51.5 \pm 3.4$ & 1.2 & $5.8 \pm 1.9$ & 0.1 & FIES \\
\hline 2011 Jan 11 & 5573.509 & 0.848 & $21.5 \pm 3.5$ & 0.4 & $43.8 \pm 2.0$ & -0.6 & FIES \\
\hline 2011 Jan 15 & 5577.505 & 0.876 & $13.8 \pm 3.4$ & -1.3 & $52.6 \pm 2.0$ & 0.3 & FIES \\
\hline 2011 Jan 15 & 5577.509 & 0.876 & $15.1 \pm 3.4$ & -0.0 & $52.6 \pm 1.9$ & 0.3 & FIES \\
\hline 2011 Jan 15 & 5577.512 & 0.876 & $14.5 \pm 3.5$ & -0.6 & $52.7 \pm 2.0$ & 0.4 & FIES \\
\hline 2011 Feb 11 & 5604.367 & 0.064 & $34.4 \pm 3.5$ & 1.2 & $28.3 \pm 2.0$ & 0.0 & FIES \\
\hline 2011 Feb 13 & 5606.027 & 0.075 & $36.1 \pm 3.4$ & -1.5 & $21.9 \pm 2.0$ & -0.6 & FEROS \\
\hline 2011 Feb 20 & 5613.359 & 0.127 & $50.3 \pm 3.3$ & 3.7 & $9.3 \pm 2.0$ & -1.3 & FIES \\
\hline 2011 Mar 22 & 5643.024 & 0.334 & $50.0 \pm 3.4$ & 0.2 & $5.8 \pm 1.9$ & -0.5 & FEROS \\
\hline 2011 Mar 27 & 5648.379 & 0.371 & $52.5 \pm 3.4$ & 3.3 & $5.1 \pm 1.9$ & -2.0 & FIES \\
\hline
\end{tabular}




\begin{tabular}{|c|c|c|c|c|c|c|c|}
\hline $\begin{array}{l}\text { Date } \\
\text { (yyyy mm dd) }\end{array}$ & $\begin{array}{c}\text { HJD } \\
(-2,450,000)\end{array}$ & $\phi$ & $\begin{array}{c}V_{r}(\mathrm{Aa}) \\
\left(\mathrm{km} \mathrm{s}^{-1}\right)\end{array}$ & $\begin{array}{c}O-C(\mathrm{Aa}) \\
\left(\mathrm{km} \mathrm{s}^{-1}\right)\end{array}$ & $\begin{array}{c}V_{r}(\mathrm{Ab}) \\
\left(\mathrm{km} \mathrm{s}^{-1}\right)\end{array}$ & $\begin{array}{c}O-C(\mathrm{Ab}) \\
\left(\mathrm{km} \mathrm{s}^{-1}\right)\end{array}$ & Instrument \\
\hline 2011 Apr 8 & 5660.353 & 0.455 & $47.1 \pm 3.4$ & -0.3 & $7.8 \pm 2.0$ & -1.8 & FIES \\
\hline 2011 Sep 7 & 5812.724 & 0.519 & $43.2 \pm 3.3$ & -2.3 & $15.2 \pm 2.0$ & 3.2 & FIES \\
\hline 2011 Sep 8 & 5813.743 & 0.526 & $43.7 \pm 3.3$ & -1.5 & $16.4 \pm 2.0$ & 4.1 & FIES \\
\hline 2011 Sep 9 & 5814.717 & 0.533 & $43.8 \pm 3.5$ & -1.2 & $14.4 \pm 2.0$ & 1.8 & FIES \\
\hline 2011 Sep 10 & 5815.726 & 0.540 & $43.2 \pm 3.5$ & -1.6 & $13.6 \pm 2.0$ & 0.6 & FIES \\
\hline 2011 Sep 11 & 5816.748 & 0.547 & $42.4 \pm 3.5$ & -2.1 & $15.1 \pm 2.0$ & 1.8 & FIES \\
\hline 2011 Sep 12 & 5817.758 & 0.554 & $41.4 \pm 3.5$ & -2.9 & $15.8 \pm 1.9$ & 2.2 & FIES \\
\hline 2011 Oct 4 & 5838.961 & 0.702 & $36.7 \pm 3.4$ & -0.2 & $27.0 \pm 1.9$ & 3.6 & HRS \\
\hline 2011 Nov 9 & 5874.575 & 0.951 & $-17.6 \pm 2.6$ & 3.7 & $101.1 \pm 1.4$ & 0.7 & HERMES \\
\hline 2011 Nov 16 & 5881.847 & 0.001 & $-82.8 \pm 1.2$ & 2.9 & $184.7 \pm 1.1$ & -1.1 & HRS \\
\hline 2011 Nov 18 & 5883.824 & 0.015 & $-29.5 \pm 2.3$ & 5.3 & $121.7 \pm 1.2$ & 3.2 & HRS \\
\hline 2011 Nov 22 & 5887.815 & 0.043 & $19.4 \pm 3.4$ & -0.3 & $46.7 \pm 2.0$ & 0.5 & HRS \\
\hline 2011 Nov 24 & 5889.821 & 0.057 & $29.8 \pm 3.6$ & -0.2 & $33.4 \pm 1.9$ & 0.8 & HRS \\
\hline 2012 Jan 3 & 5929.710 & 0.336 & $52.0 \pm 3.6$ & 2.2 & $5.6 \pm 1.9$ & -0.8 & HRS \\
\hline 2012 Apr 5 & 6023.388 & 0.990 & $-86.4 \pm 1.8$ & 1.4 & $185.6 \pm 1.0$ & -3.0 & HERMES \\
\hline 2012 Apr 5 & 6023.393 & 0.990 & $-87.4 \pm 2.0$ & 0.5 & $185.9 \pm 1.1$ & -2.8 & HERMES \\
\hline 2012 Apr 6 & 6024.329 & 0.996 & $-94.3 \pm 1.8$ & -1.1 & $193.4 \pm 1.4$ & -2.3 & HERMES \\
\hline 2012 Apr 6 & 6024.333 & 0.997 & $-93.1 \pm 1.2$ & 0.1 & $192.9 \pm 1.1$ & -2.8 & HERMES \\
\hline 2012 Apr 6 & 6024.344 & 0.997 & $-95.6 \pm 1.4$ & -2.5 & $196.4 \pm 1.3$ & 0.7 & CAFÉ \\
\hline 2012 Apr 7 & 6025.314 & 0.003 & $-80.7 \pm 1.3$ & -0.5 & $178.3 \pm 1.2$ & -0.2 & CAFÉ \\
\hline 2012 Apr 8 & 6026.321 & 0.010 & $-52.2 \pm 1.6$ & 1.1 & $142.9 \pm 0.9$ & -0.1 & CAFÉ \\
\hline 2012 Apr 8 & 6026.326 & 0.010 & $-53.6 \pm 1.8$ & -0.4 & $145.0 \pm 0.9$ & 2.2 & HERMES \\
\hline 2012 Oct 26 & 6226.679 & 0.410 & $46.5 \pm 3.3$ & -2.0 & $9.5 \pm 1.9$ & 1.4 & HERMES \\
\hline 2012 Oct 27 & 6227.659 & 0.416 & $44.6 \pm 3.5$ & -3.7 & $12.2 \pm 1.9$ & 3.9 & HERMES \\
\hline 2012 Oct 29 & 6229.584 & 0.430 & $44.7 \pm 3.5$ & -3.3 & $11.5 \pm 1.9$ & 2.8 & HERMES \\
\hline 2012 Dec 23 & 6285.551 & 0.821 & $25.2 \pm 3.5$ & -0.3 & $38.7 \pm 2.0$ & 0.2 & FIES \\
\hline 2012 Dec 24 & 6286.599 & 0.828 & $26.1 \pm 3.5$ & 1.7 & $35.4 \pm 2.0$ & -4.5 & FIES \\
\hline 2012 Dec 25 & 6287.467 & 0.834 & $23.0 \pm 3.5$ & -0.5 & $37.1 \pm 2.0$ & -4.1 & FIES \\
\hline 2013 Jan 28 & 6321.409 & 0.071 & $36.1 \pm 3.5$ & -0.1 & $24.4 \pm 2.0$ & 0.0 & FIES \\
\hline 2013 Jan 29 & 6322.470 & 0.079 & $40.2 \pm 3.5$ & 1.6 & $21.7 \pm 2.0$ & 0.5 & FIES \\
\hline 2013 Jan 30 & 6323.396 & 0.085 & $38.8 \pm 3.4$ & -1.5 & $19.6 \pm 2.0$ & 0.6 & FIES \\
\hline 2013 Feb 5 & 6329.389 & 0.127 & $47.1 \pm 3.5$ & 0.5 & $13.6 \pm 1.9$ & 3.0 & FIES \\
\hline 2013 Feb 5 & 6329.391 & 0.127 & $48.2 \pm 3.5$ & 1.6 & $11.4 \pm 1.9$ & 0.8 & FIES \\
\hline 2013 Feb 15 & 6339.330 & 0.196 & $50.3 \pm 3.4$ & 0.4 & $3.5 \pm 1.9$ & -2.7 & FIES \\
\hline 2013 Mar 9 & 6361.470 & 0.351 & $49.2 \pm 3.5$ & -0.3 & $5.1 \pm 1.9$ & -1.5 & HERMES \\
\hline 2013 Mar 9 & 6361.473 & 0.351 & $49.2 \pm 3.5$ & -0.3 & $5.0 \pm 1.9$ & -1.6 & HERMES \\
\hline 2013 Mar 10 & 6362.459 & 0.358 & $49.9 \pm 3.4$ & 0.5 & $4.5 \pm 1.9$ & -2.3 & HERMES \\
\hline 2013 Oct 25 & 6590.638 & 0.951 & $-18.9 \pm 2.6$ & 2.9 & $103.3 \pm 1.3$ & 2.2 & HERMES \\
\hline 2013 Oct 26 & 6591.627 & 0.958 & $-28.7 \pm 2.3$ & 0.6 & $114.2 \pm 1.3$ & 3.1 & HERMES \\
\hline 2013 Oct 27 & 6592.627 & 0.965 & $-36.2 \pm 2.2$ & 2.5 & $124.8 \pm 1.2$ & 1.3 & HERMES \\
\hline 2013 Oct 28 & 6593.585 & 0.972 & $-52.2 \pm 1.3$ & -2.5 & $138.0 \pm 0.9$ & -0.2 & HERMES \\
\hline 2013 Oct 29 & 6594.697 & 0.980 & $-65.0 \pm 1.4$ & 0.5 & $160.4 \pm 1.0$ & 1.4 & HERMES \\
\hline 2013 Oct 30 & 6595.768 & 0.987 & $-83.3 \pm 1.4$ & -1.1 & $180.6 \pm 1.4$ & -0.6 & HERMES \\
\hline 2013 Oct 31 & 6596.596 & 0.993 & $-93.4 \pm 1.3$ & -1.5 & $197.1 \pm 1.3$ & 3.0 & HERMES \\
\hline 2013 Oct 31 & 6596.777 & 0.994 & $-93.5 \pm 1.4$ & -0.6 & $197.9 \pm 1.2$ & 2.5 & HERMES \\
\hline 2013 Nov 1 & 6597.783 & 0.001 & $-88.9 \pm 1.3$ & -2.3 & $188.2 \pm 1.1$ & 1.2 & HERMES \\
\hline 2013 Nov 2 & 6598.787 & 0.008 & $-60.9 \pm 1.6$ & 1.5 & $157.0 \pm 0.9$ & 2.0 & HERMES \\
\hline 2013 Nov 4 & 6600.789 & 0.022 & $-13.7 \pm 2.8$ & 0.5 & $92.2 \pm 1.6$ & 1.1 & HERMES \\
\hline 2013 Nov 5 & 6601.787 & 0.029 & $0.3 \pm 3.3$ & -0.7 & $71.1 \pm 1.9$ & 0.1 & HERMES \\
\hline 2013 Nov 6 & 6602.797 & 0.036 & $13.6 \pm 3.2$ & 1.8 & $52.9 \pm 1.9$ & -3.7 & HERMES \\
\hline 2013 Nov 7 & 6603.788 & 0.043 & $18.9 \pm 3.4$ & -0.7 & $45.5 \pm 1.9$ & -0.9 & HERMES \\
\hline 2013 Nov 8 & 6604.798 & 0.050 & $23.1 \pm 3.4$ & -2.3 & $39.8 \pm 1.9$ & 1.2 & HERMES \\
\hline 2013 Nov 9 & 6605.800 & 0.057 & $27.6 \pm 3.4$ & -2.3 & $33.0 \pm 1.9$ & 0.3 & HERMES \\
\hline 2013 Nov 10 & 6606.798 & 0.064 & $31.0 \pm 3.5$ & -2.4 & $28.5 \pm 1.9$ & 0.4 & HERMES \\
\hline 2013 Nov 11 & 6607.777 & 0.071 & $32.8 \pm 3.3$ & -3.3 & $25.5 \pm 1.9$ & 1.0 & HERMES \\
\hline 2013 Nov 29 & 6626.607 & 0.202 & $51.2 \pm 3.5$ & 1.2 & $5.5 \pm 1.9$ & -0.5 & CAFÉ \\
\hline 2014 Jan 11 & 6669.455 & 0.502 & $45.5 \pm 3.5$ & -0.5 & $11.9 \pm 1.9$ & 0.6 & CAFÉ \\
\hline 2014 Mar 10 & 6727.368 & 0.906 & $2.4 \pm 3.4$ & -3.5 & $66.2 \pm 1.8$ & 1.7 & CAFÉ \\
\hline 2014 Mar 11 & 6728.376 & 0.913 & $-0.8 \pm 3.4$ & -3.8 & $68.6 \pm 1.9$ & 0.3 & CAFÉ \\
\hline 2014 Mar 20 & 6737.401 & 0.976 & $-60.1 \pm 1.8$ & -2.0 & $150.0 \pm 0.9$ & 0.7 & CAFÉ \\
\hline 2014 Mar 21 & 6738.308 & 0.982 & $-72.7 \pm 1.5$ & -0.8 & $167.1 \pm 1.1$ & -0.5 & CAFÉ \\
\hline 2014 Mar 22 & 6739.285 & 0.989 & $-87.0 \pm 1.5$ & -0.3 & $185.7 \pm 1.3$ & -1.4 & CAFÉ \\
\hline 2014 Mar 23 & 6740.290 & 0.996 & $-96.3 \pm 1.1$ & -3.0 & $194.3 \pm 1.4$ & -1.5 & CAFÉ \\
\hline
\end{tabular}




\section{REFERENCES}

Abergel, A., Teyssier, D., Bernard, J. P., et al. 2003, A\&A, 410, 577 Aceituno, J., Sánchez, S. F., Grupp, F., et al. 2013, A\&A, 552, A31 Barbá, R. H., Gamen, R., Arias, J. I., et al. 2010, RMxAA, 38, 30

Béjar, V. J. S., Zapatero Osorio, M. R., Rebolo, R., et al. 1999, ApJ, 521,671

Berghoefer, T. W., \& Schmitt, J. H. M. M. 1994, A\&A, 290, 435

Bolton, C. T. 1974, ApJL, 192, L7

Bouy, H., Huélamo, N., Martín, E. L., et al. 2009, A\&A, 493, 931

Bowler, B. P., Waller, W. H., Megeath, S. T., Patten, B. M., \& Tamura, M. 2009, AJ, 137, 3685

Braun, H., \& Langer, N. 1995, A\&A, 297, 483

Brott, I., de Mink, S. E., Cantiello, M., et al. 2011, A\&A, 530, A115

Brown, A. G. A., de Geus, E. J., \& de Zeeuw, P. T. 1994, A\&A, 289, 101

Burnham, S. W. 1892, AN, 130, 257

Caballero, J. A. 2005, AN, 326, 1007

Caballero, J. A. 2007, A\&A, 466, 917

Caballero, J. A. 2008a, MNRAS, 383, 750

Caballero, J. A. 2008b, A\&A, 487, 667

Caballero, J. A. 2013, Star Formation Newsl., 243, 6

Caballero, J. A. 2014, Obs, 134, 273

Caballero, J. A., Albacete-Colombo, J. F., \& López-Santiago, J. 2010, A\&A, 521, A 45

Caballero, J. A., \& Dinis, L. 2008, AN, 329, 801

Caballero, J. A., López-Santiago, J., de Castro, E., \& Cornide, M. 2009, AJ, 137,5012

Compiègne, M., Abergel, A., Verstraete, L., et al. 2007, A\&A, 471, 205

de Zeeuw, P. T., Hoogerwerf, R., de Bruijne, J. H. J., Brown, A. G. A., \& Blaauw, A. 1999, AJ, 117, 354

Dray, L. M., \& Tout, C. A. 2007, MNRAS, 376, 61

Ducati, J. R., Bevilacqua, C. M., Rembold, S. B., \& Ribeiro, D. 2001, ApJ, 558,309

Edwards, T. W. 1976, AJ, 81, 245

Ekström, S., Georgy, C., Eggenberger, P., et al. 2012, A\&A, 537, A146

Etzel, P. B. 2004, SBOP: Spectroscopic Binary Orbit Program (San Diego State University)

Frost, E. B., \& Adams, W. S. 1904, ApJ, 19, 151

Garrison, R. F. 1967, PASP, 79, 433

Goicoechea, J. R., Pety, J., Gerin, M., et al. 2006, A\&A, 456, 565

Goicoechea, J. R., Pety, J., Gerin, M., Hily-Blant, P., \& Le Bourlot, J. 2009, A\&A, 498, 771

González Hernández, J. I., Caballero, J. A., Rebolo, R., et al. 2008, A\&A, 490, 1135

Groote, D., \& Hunger, K. 1997, A\&A, 319, 250

Habart, E., Abergel, A., Walmsley, C. M., Teyssier, D., \& Pety, J. 2005, A\&A, 437, 177

Hartkopf, W. I., Mason, B. D., \& McAlister, H. A. 1996, AJ, 111, 370

Hernández, J., Calvet, N., Hartmann, L., et al. 2005, AJ, 129, 856

Herrero, A., Kudritzki, R. P., Vilchez, J. M., et al. 1992, A\&A, 261, 209

Herrero, A., Puls, J., \& Najarro, F. 2002, A\&A, 396, 949

Hodapp, K. W., Iserlohe, C., Stecklum, B., \& Krabbe, A. 2009, ApJL, 701, L100

Horch, E., Ninkov, Z., \& Franz, O. G. 2001, AJ, 121, 1583

Horch, E. P., Meyer, R. D., \& van Altena, W. F. 2004, AJ, 127, 1727

Hormuth, F., Hippler, S., Brandner, W., Wagner, K., \& Henning, T. 2008, Proc. SPIE, 7014, 701448

Hummel, C. A., Zavala, R. T., \& Sanborn, J. 2013, CEAB, 37, 127

Johnson, H. L., Mitchell, R. I., Iriarte, B., \& Wisniewski, W. Z. 1966, CoLPL, 4,99

Kaufer, A., Stahl, O., Tubbesing, S., et al. 1999, Msngr, 95, 8

Landstreet, J. D., \& Borra, E. F. 1978, ApJL, 224, L5

Lee, T. A. 1968, ApJ, 152, 913

Lutz, T. E., \& Kelker, D. H. 1973, PASP, 85, 573

Maíz-Apellániz, J. 2001, AJ, 121, 2737

Maíz-Apellániz, J. 2004, PASP, 116, 859

Maíz-Apellániz, J. 2005a, PASP, 117, 615

Maíz Apellániz, J. 2005b, in The Three-dimensional Universe with Gaia, ed. C. Turon, K. S. O'Flaherty, \& M. A. C. Perryman (ESA-SP 576; Noordwijk: ESA), 179

Maíz Apellániz, J. 2006, AJ, 131, 1184

Maíz Apellániz, J. 2007, in ASP Conf. Ser. 364, The Future of Photometric, Spectrophotometric and Polarimetric Standardization, ed. C. Sterken (San Francisco, CA: ASP), 227

Maíz Apellániz, J. 2010, A\&A, 518, A1
Maíz Apellániz, J. 2013a, in Highlights of Spanish Astrophysics VII, ed. J. C. Guirado, L. M. Lara, V. Quilis, \& J. Gorgas (New York: Springer)

Maíz Apellániz, J. 2013b, in Highlights of Spanish Astrophysics VII, ed. J. C. Guirado, L. M. Lara, V. Quilis, \& J. Gorgas (New York: Springer)

Maíz Apellániz, J., Evans, C. J., Barbá, R. H., et al. 2014, A\&A, 564, A63

Maíz Apellániz, J., Pellerin, A., Barbá, R. H., et al. 2012, in ASP Conf. Ser. 465, Proceedings of a Scientific Meeting in Honor of Anthony, ed. L. Drissen, N. St-Louis, C. Robert, \& A. F. J. Moffat (San Francisco, CA: ASP), 484

Maíz-Apellániz, J., Walborn, N. R., Galué, H. Á., \& Wei, L. H. 2004, ApJS, 151,103

Mayne, N. J., \& Naylor, T. 2008, MNRAS, 386, 261

Miczaika, G. R. 1950, ApJ, 111, 443

Morrell, N., \& Levato, H. 1991, ApJS, 75, 965

Najarro, F., Hanson, M. M., \& Puls, J. 2011, A\&A, 535, A32

Naylor, T. 2009, MNRAS, 399, 432

Negueruela, I., Maíz Apellániz, J., Simón-Díaz, S., et al. 2014, in Highlights of Spanish Astrophysics VIII, Proć. of the Xi Scientific Meeting of the Spanish Astronomical Society, e-print (arXiv:1412.5638)

Nieva, M.-F., \& Simón-Díaz, S. 2011, A\&A, 532, A2

Ogura, K., \& Sugitani, K. 1998, PASA, 15, 91

Perryman, M. A. C., Lindegren, L., Kovalevsky, J., et al. 1997, A\&A, 323, L49

Pety, J., Teyssier, D., Fossé, D., et al. 2005, A\&A, 435, 885

Pound, M. W., Reipurth, B., \& Bally, J. 2003, AJ, 125, 2108

Raskin, G., van Winckel, H., Hensberge, H., et al. 2011, A\&A, 526, A69

Repolust, T., Puls, J., \& Herrero, A. 2004, A\&A, 415, 349

Rimmer, P. B., Herbst, E., Morata, O., \& Roueff, E. 2012, A\&A, 537, A7

Rivilla, V. M., Martín-Pintado, J., Jiménez-Serra, I., \& Rodríguez-Franco, A. 2013, A\&A, 554, A48

Sacco, G. G., Franciosini, E., Randich, S., \& Pallavicini, R. 2008, A\&A, 488,167

Salpeter, E. E. 1955, ApJ, 121, 161

Sanz-Forcada, J., Franciosini, E., \& Pallavicini, R. 2004, A\&A, 421, 715

Schaefer, G. H. 2013, in Setting a New Standard in the Analysis of Binary Stars, ed. K. Pavlovski, A. Tkachenko, \& G. Torres (ESA-SP 64; Noordwijk: ESA), 181

Schneider, F. R. N., Izzard, R. G., de Mink, S. E., et al. 2014a, ApJ, 780, 117

Schneider, F. R. N., Langer, N., de Koter, A., et al. 2014b, A\&A, 570, A66

Sherry, W. H., Walter, F. M., \& Wolk, S. J. 2004, AJ, 128, 2316

Sherry, W. H., Walter, F. M., Wolk, S. J., \& Adams, N. R. 2008, AJ, 135, 1616

Simón-Díaz, S. 2010, A\&A, 510, A22

Simón-Díaz, S., Caballero, J. A., \& Lorenzo, J. 2011a, ApJ, 742, 55

Simón-Díaz, S., Castro, N., Garcia, M., Herrero, A., \& Markova, N. 2011b, BSRSL, 80, 514

Simón-Díaz, S., Castro, N., Herrero, A., et al. 2011c, JPhCS, 328, 012021

Simón-Díaz, S., Garcia, M., Herrero, A., Maíz Apellániz, J., \& Negueruela, I. 2011d, in Stellar Clusters \& Associations: A RIA Workshop on Gaia, ed. E. J. Alfaro Navarro, A. T. Gallego Calvente, \& M. R. Zapatero Osorio, 255

Simón-Díaz, S., \& Herrero, A. 2014, A\&A, 562, A135

Skinner, S. L., Sokal, K. R., Cohen, D. H., et al. 2008, ApJ, 683, 796

Stickland, D. J., \& Lloyd, C. 2001, Obs, 121, 1

Telting, J. H., Ávila, G., Buchhave, L., et al. 2014, AN, 335, 41

ten Brummelaar, T., Mason, B. D., McAlister, H. A., et al. 2000, AJ, 119, 2403

Tokovinin, A. 2008, MNRAS, 389, 925

Townsend, R. H. D., Rivinius, T., Rowe, J. F., et al. 2013, ApJ, 769, 33

Tull, R. G. 1998, Proc. SPIE, 3355, 387

Turner, N. H., ten Brummelaar, T. A., Roberts, L. C., et al. 2008, AJ, 136, 554

van Bever, J., \& Vanbeveren, D. 1998, A\&A, 334, 21

van Leeuwen, F. 2007, A\&A, 474, 653

van Loon, J. T., \& Oliveira, J. M. 2003, A\&A, 405, L33

Vogt, N. 1976, A\&A, 53, 9

Walborn, N. R. 1974, ApJL, 191, L95

Walter, F. M., Sherry, W. H., Wolk, S. J., \& Adams, N. R. 2008, in Handbook of Star Forming Regions, Volume I: The Northern Sky ASP Monograph Publications, Vol. 4., ed. B. Reipurth (San Francisco, CA: ASP), 732

Ward-Thompson, D., Nutter, D., Bontemps, S., Whitworth, A., \& Attwood, R. 2006, MNRAS, 369, 1201

Whitworth, A., Bate, M. R., Nordlund, Å., Reipurth, B., \& Zinnecker, H. 2007, in Protostars and Planets V, ed. B. Reipurth, D. Jewitt, \& K. Keil (Tucson, AZ: Univ. Arizona Press), 459

Wolk, S. J. 1996, PhD thesis, State Univ. New York

Zapatero Osorio, M. R., Béjar, V. J. S., Pavlenko, Y., et al. 2002, A\&A, 384,937

Zinnecker, H., \& Yorke, H. W. 2007, ARA\&A, 45, 481 